\title{
La domestication de l'abeille par le territoire
}

Un exemple d'apiculture holiste dans le sud marocain

The Taming of the bee by the territory. An example of holistic beekeeping in the

Moroccan South

Romain Simenel, Antonin Adam, Audrey Crousilles, Lahoucine Amzil et Yildiz Aumeeruddy-Thomas

\section{(2) OpenEdition \\ Journals}

\section{Édition électronique}

URL : https://journals.openedition.org/tc/7516

DOI : $10.4000 /$ tc. 7516

ISBN : 0248-6016

ISSN : 1952-420X

Éditeur

Éditions de l'EHESS

\section{Édition imprimée}

Date de publication : 1 mai 2015

Pagination : 258-279

ISBN : 9782713224874

ISSN : 0248-6016

Référence électronique

Romain Simenel, Antonin Adam, Audrey Crousilles, Lahoucine Amzil et Yildiz Aumeeruddy-Thomas, «La domestication de l'abeille par le territoire», Techniques \& Culture [En ligne], 63 | 2015, mis en ligne le 22 décembre 2018, consulté le 29 septembre 2022. URL : http://journals.openedition.org/tc/7516 DOI : https://doi.org/10.4000/tc.7516 


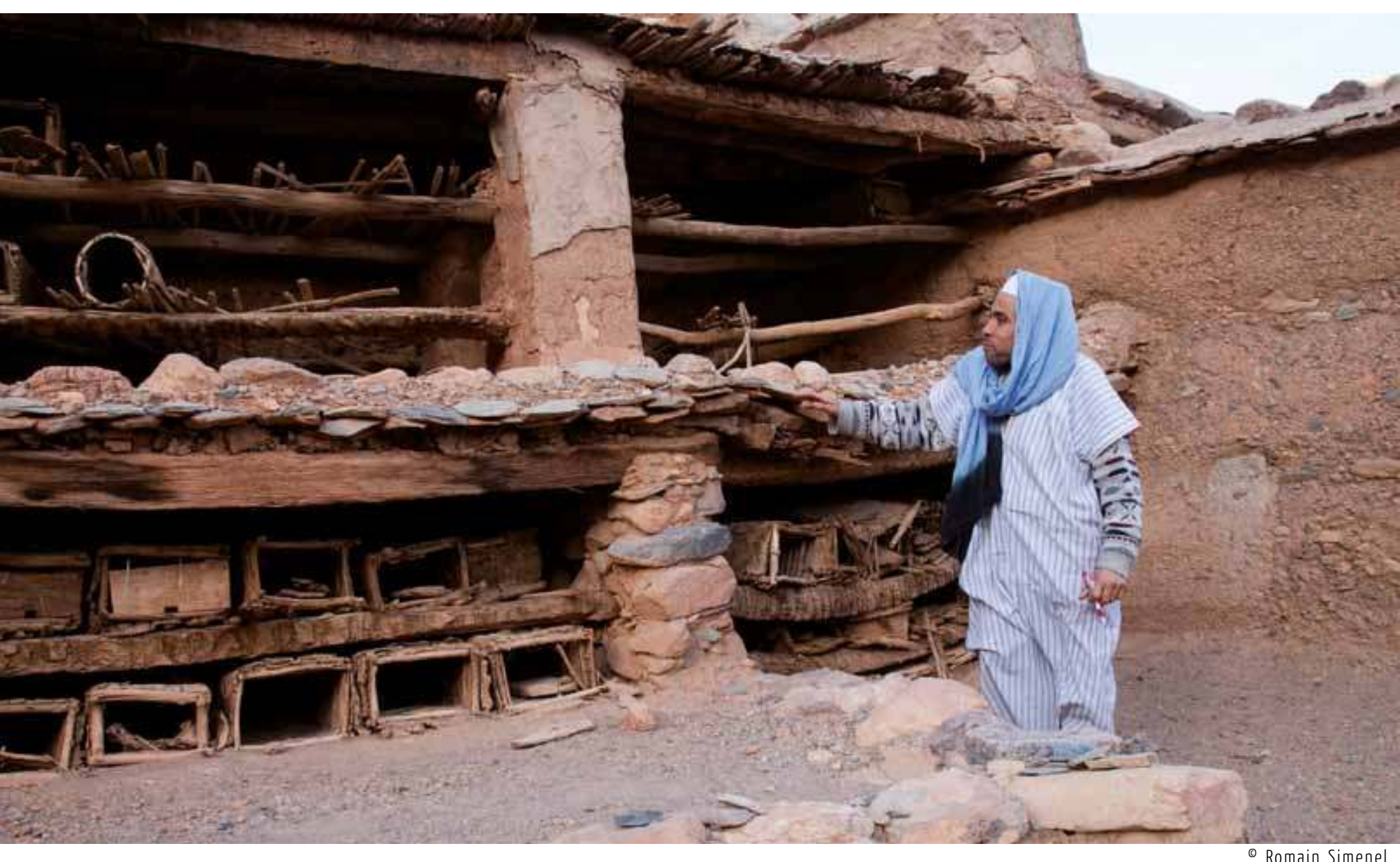




\section{LA DOMESTICATION DE L'ABELLLE PAR LE TERRITOIRE}

\section{Un exemple d’apiculture holiste dans le Sud marocain}

La problématique de l'effondrement des populations d'abeilles dans le monde ne se pose pas en des termes similaires dans les zones tempérées et dans les zones semiarides et arides. Dans ces dernières, l'effondrement est chronique et dû à des régimes de précipitations annuelles très variables qui imposent des fluctuations du niveau des populations d'abeilles. Dans ces conditions, la continuité du rapport homme-abeille ne repose pas tant sur la stabilité de la population ou du stock de pollen et nectar disponible que sur la permanence de la relation, tant pratique que symbolique ${ }^{1}$. Une telle distinction de contextes apicoles invite à préciser le régime de relation qui permet sa permanence en dehors de toute stabilité démographique d'un des acteurs de la relation, l'abeille.

La question de la domestication des abeilles ne cesse de faire débat depuis des décennies, chez les anthropologues, historiens et archéozoologues notamment, pour faire la part entre l'imaginaire du sens commun, suscité de manière universelle par la «sociabilité » remarquable de ces populations d'insectes, et l'impact des pratiques concrètes des apiculteurs sur ces dernières. Car une question dérange sans cesse du point de vue de la formulation: comment parler de domestication de l'abeille alors que celle-ci vit déjà en société et que son passage à «l'état » domestique ne change en rien son organisation sociale? Même si la langue française retient la connotation végétale du verbe « cultiver » pour qualifier le champ d'activité lié à l'exploitation des abeilles, ou celui d'« élever » pour désigner l'activité de sélection des reines, certains auteurs imputent à juste titre à ces appellations des points de vue culturels qui ne témoignent pas de la réalité du rapport technique au monde des abeilles, mais des représentations que l'on s'en fait (Marchenay, 1993; Tétart 2001). 

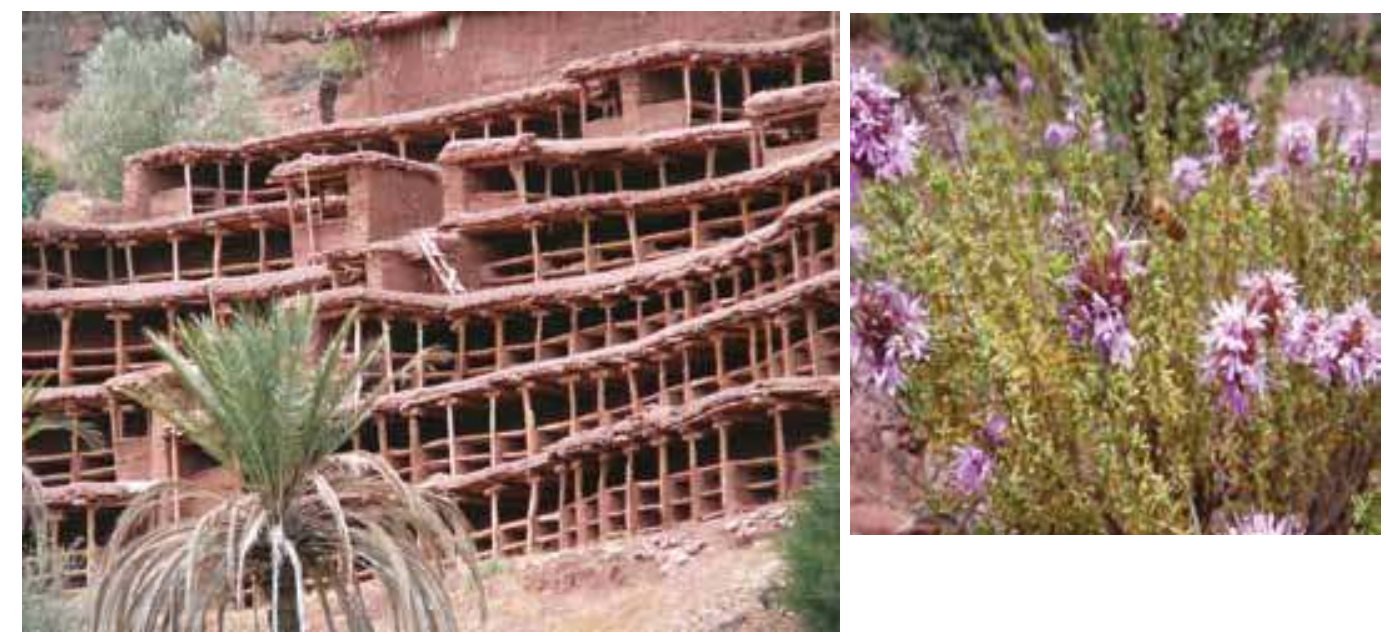

Ruchers

collectifs pour

le miel de thym.

Tazuknit.

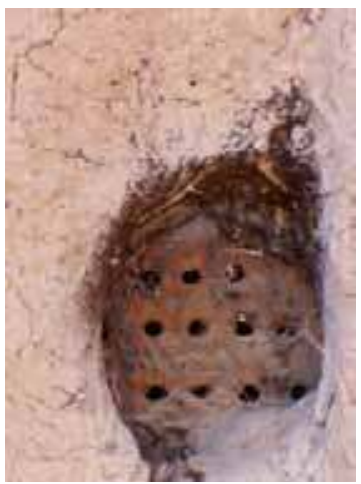

Ruche en poterie

incrustée

dans le mur.
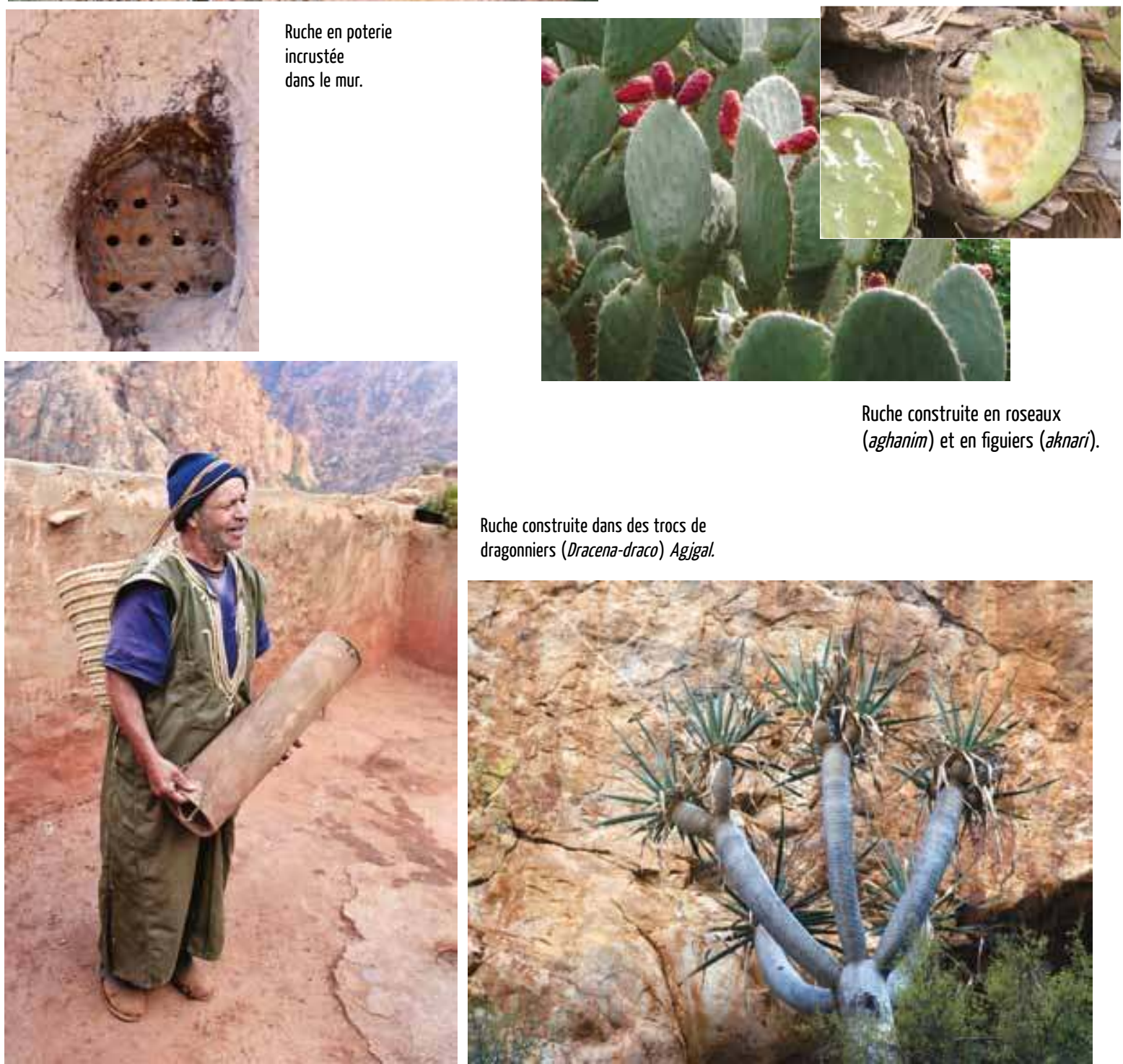

Ruche construite en roseaux

(aghanim) et en figuiers (aknari).

Ruche construite dans des trocs de

dragonniers (Dracena-draco) Agjgal.

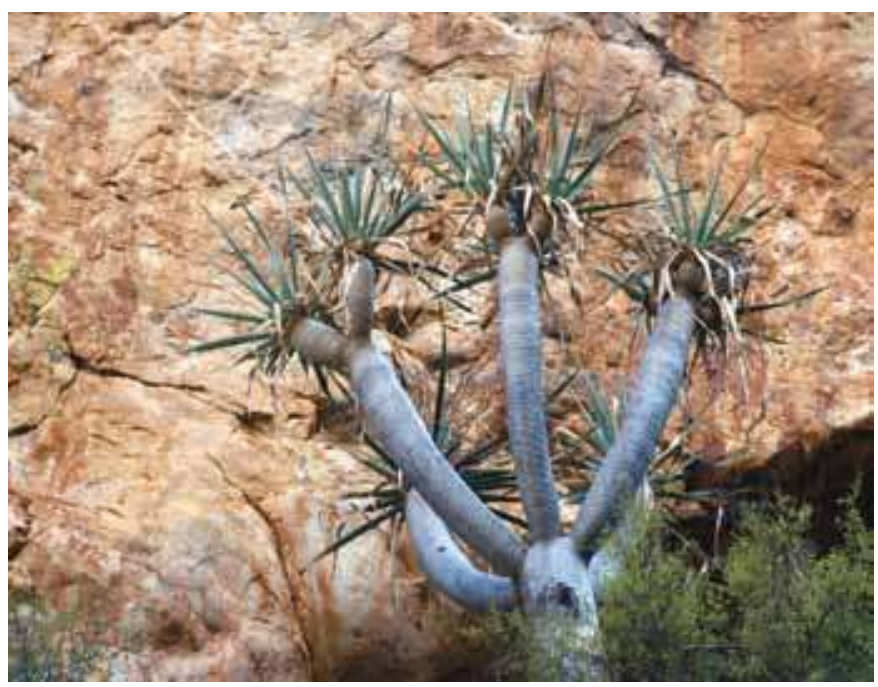

Miel d’arganiers, de jujubiers, de chardons, d'euphorbes diverses et variées, de thym, autant de miels dont la production dépend de la diversité de savoir-faire locaux qui prend forme dans la variété des types de ruches traditionnelles, allant des ruches en troncs de dragonnier aux ruches en poterie. 
Le paradoxe de la domestication des abeilles est ainsi soulevé de manière légitime, car selon les spécialistes, dans les faits, il n'y a véritablement domestication, au sens technique du terme, qu'à partir de l'apiculture moderne (Tétart, 2001 : 180). Deux arguments soutiennent cette proposition. Le premier consiste à dire que les abeilles restent en relation avec leur milieu naturel et sont donc entretenues dans une situation semblable à celle où elles se trouvent (Tétart, 2001 : 181). Reste à savoir ce que l'on entend par «milieu naturel » des abeilles, s'agit-il de l'habitat, des structures en cire, de la place dans le territoire, de l'écosystème environnant? Le second s'appuie sur le constat que « dans le domaine de la domestication animale, ce terme (élevage) sous-entend une intervention de l'homme au niveau de la reproduction sexuée, ce qui implique un contrôle direct sur les fonctions vitales de l'animal » (Tétart, 2001 : 179). Là encore, face au manque de données sur les pratiques des apiculteurs traditionnels ${ }^{2}$ du monde entier, il paraît difficile d'identifier à l'avance où et comment se jouerait une telle intervention humaine sur le peuple des abeilles. Et puis, faut-il encore s'accorder sur ce à quoi se limite le « contrôle direct ». Aux opérations techniques répétées de manipulation de l'abeille par l'apiculture moderne dont l'effet ne se fait jamais ressentir à plus d'une génération? Mais l'apiculteur traditionnel n'a-t-il pas un impact plus important et plus durable sur les abeilles, leur comportement et leur métabolisme, en ayant façonné, structuré, et rythmé leur milieu et leur espace de vie?

En cherchant à requalifier la domestication des abeilles à partir de pratiques plus holistes et plus ancrées dans le territoire, notre propos est d'interroger à partir d'un cas ethnographique précis, localisé dans le Sud ouest marocain (D'Essaouira à Tan Tan), la manière dont les apiculteurs de ces régions intègrent idéologiquement et techniquement les abeilles dans leur monde. Une telle domestication s'appuie autant sur l'aménagement du territoire que sur des interventions techniques, et résulte du concours entre des pratiques de territorialisation de la ruche et des gestes et savoirs venant ponctuer le parcours de l'essaim. Même si la pratique de l'apiculture est pour la plupart du temps individuelle et masculine, les pratiques apicoles se recoupent pour former un corpus commun qui ne cesse de s'enrichir d'innovations personnelles.

Même si cela peut paraître paradoxal selon les a priori du déterminisme climatique et géographique, ce n'est pas au Nord mais bien dans les régions semi-arides du Sud ouest du Maroc que l'on trouve la plus grande diversité de miel du Maghreb. Mais où se cache la raison expliquant que ces territoires soient plus enclins à l'apiculture que d'autres moins arides plus au Nord? Quelle est la relation entre les apiculteurs du Sud marocain et les abeilles capable d'expliquer un tel exploit? Dans ces régions semi-arides bénéficiant certes d'un apport d'humidité océanique, le foisonnement des pollens et nectars, des essaims et des savoir-faire apicoles, ainsi que la spécificité biologique de l'abeille saharienne, résultent en grande partie de la coévolution entre les groupes berbères du sud du Maroc et un écosystème forestier unique, l'arganeraie. L'agroécosystème façonné par les générations de paysans chleuhs (de langue tachelhit) s'appuie essentiellement sur l'arganier (Argania spinosa L.), espèce endémique couvrant environ 850000 hectares et permettant le développement d'un couvert floristique mellifère diversifié. Cette forêt clairsemée est habitée, transformée et façonnée par de nombreux groupes berbères, tels que les Haha (province d'Essaouira), les Ait Ba’amran (province de Sidi Ifni) ou les Ida ou Tanan (province d'Agadir), afin de répondre à leurs besoins agropastoraux, dont ceux liés à l'apiculture.

Les savoir-faire des apiculteurs de ces régions reposent sur l'aménagement de différents types d'espaces agraires, l'apiculture faisant partie intégrante de l'agro-écosystème global. 
Bien que sédentaire, l'apiculture dans le sud du Maroc implique une grande diversité d'espaces, comprenant les falaises à chutes d'eau, les champs de céréales et d'arganiers, les zones arboricoles fermées, les zones de monoculture de figuiers de barbarie (Opuntia ficus indica (L.) Mill.), les parcours dominés par les euphorbes (Euphorbia officinarum Hook.f. \& Coss. (subsp. officinarum et subsp. echinus), Euphorbia balsamifera Aiton) et les arganiers. Chaque unité spatiale du territoire rural est le support d'un certain degré de domestication des abeilles: capture des essaims sauvages, acclimatation des ruches, production de miel, reproduction des essaims. Les savoir-faire des apiculteurs s'illustrent par la connaissance sur le comportement des abeilles (comportement alimentaire, temporalité des préférences de matières apicoles), leur métabolisme (vertus des plantes sur la vie des abeilles, leur capacité à essaimer, leur capacité à nettoyer la ruche), et la pollinisation qu'elles permettent. Les apiculteurs du Sud ouest marocain jouent ainsi sur la pluralité des espaces de cultures et des espèces qui y poussent pour intégrer l'abeille dans le territoire rural de manière holiste. Ils adaptent l'abeille au territoire tout en adaptant le territoire à l'abeille. Ce faisant, l'apiculteur dispose de plusieurs cordes à son arc pour préserver ses essaims des maladies et leur apporter la nourriture dont ils ont besoin.

\section{Méthodologie}

Les matériaux présentés dans cet article ont été recueillis lors de nombreux séjours de terrain entre 2008 et 2012 dans les régions d'Essaouira, d'Agadir, de Sidi Ifni, et de Guelmim $^{3}$. Les trois groupes tribaux chez qui les entretiens se sont déroulés sont les Haha, les Ida ou Tanan et les Ait Ba'amran, trois ensembles parlant le tachelhit, dialecte berbère local. La plupart des entretiens avec les apiculteurs ont été réalisés en tachelhit ou en arabe dialectal, soit directement par les auteurs berbérophones de l'article, soit par l'intermédiaire d'un interprète. Ils ont tous été réalisés in situ près des ruches lors des activités apicoles, permettant de manière coordonnée l'observation des pratiques associées au discours. Nous avons notamment pu bénéficier de l'expérience et du savoir des apiculteurs Lahoucine Bounnit, Mohamed Sforge et Salah Sayed.

\section{De la gauche vers la droite: comment domestiquer les abeilles par un simple déplacement}

Dans la cosmogonie berbère du Sud marocain, les abeilles (tizoi) sont sacrées. Elles incarnent idéalement les valeurs de sociabilité, d'honnêteté et de droiture. Elles sont censées produire du miel exclusivement pour le prophète Mohamed. Aussi, comme le raconte la tradition orale, c'est parce que les hommes leur ont caché la mort du prophète, que les abeilles continuent à produire du miel (tammant). Ainsi, la conception dominante sur les abeilles veut que celles-ci soient déjà domestiquées par l'entremise de la baraka divine. Malgré la prégnance du discours religieux qui tend à définir le peuple abeille de manière uniforme comme une espèce déjà « socialisée », les apiculteurs du Sud marocain distinguent clairement les abeilles non soumises aux pratiques humaines de celles exploitées dans l'apiculture traditionnelle. Tout comme pour les autres animaux soumis à 
la domestication et notamment à l'élevage en monde berbère, les abeilles des apiculteurs ont leurs analogues sylvestres. Le système analogique de représentation en vigueur dans cette aire culturelle impose un dualisme entre toute espèce domestiquée par l'homme, et son pendant sylvestre non domestiqué, qu'il y ait ou non une affiliation d'espèce à espèce reconnue (Simenel, 2010 : 171). Le sanglier est ainsi la vache de la forêt, la perdrix est la poule de la forêt, et la gazelle la chèvre. De même, on oppose systématiquement les abeilles de la gauche qui vivent dans le milieu sylvestre, à celles de la droite, qui sont exploitées dans les ruches. Ces dernières sont considérées comme des habitats d'animaux d'élevage, des bergeries que l'on baptise du nom de la famille propriétaire, scellant ainsi leur appropriation domestique. La distinction entre abeilles sauvage et domestique s'appuie aussi sur une différenciation des physicalités de ces deux catégories d'abeilles. Les premières sont ainsi qualifiées de « sourdes » alors que les secondes seraient dotées de l'ouie. Par ce jeu de représentations, le territoire apicole se polarise en fonction d'une orientation gauche (forêt)/droite (espace cultivé), typique d'une césure plus générale dans la culture berbère entre le monde des jnoun situé à gauche, le côté interdit du monde (haram), et le monde des humains situé à droite, le côté licite du monde (halal).

Cependant, en réalité l'apiculteur s'appuie sur un flot continuel d'abeilles allant et venant de la forêt vers les espaces cultivés et vice versa. Les deux pôles du monde des abeilles s'avèrent dans la pratique apicole bien évidemment totalement reliés, puisque l'apiculteur se fournit à la base en essaims provenant de la forêt et que les essaims fraîchement sortis des ruches ont souvent tendance à retourner en forêt. Les abeilles de la gauche passent ainsi à droite et vice versa. L'étape de l'essaimage ${ }^{4}$, lors de laquelle de nouveaux essaims se perdent et s'accrochent aux branches d'arbres dans les champs ou les forêts, nécessite une ouverture totale du territoire et une coopération entre voisins.
Le passage d'une ruche aux galettes perpendiculaires à celle aux galettes parallèles n'est pas direct, s'intercale souvent une étape intermédiaire, celle des galettes obliques.

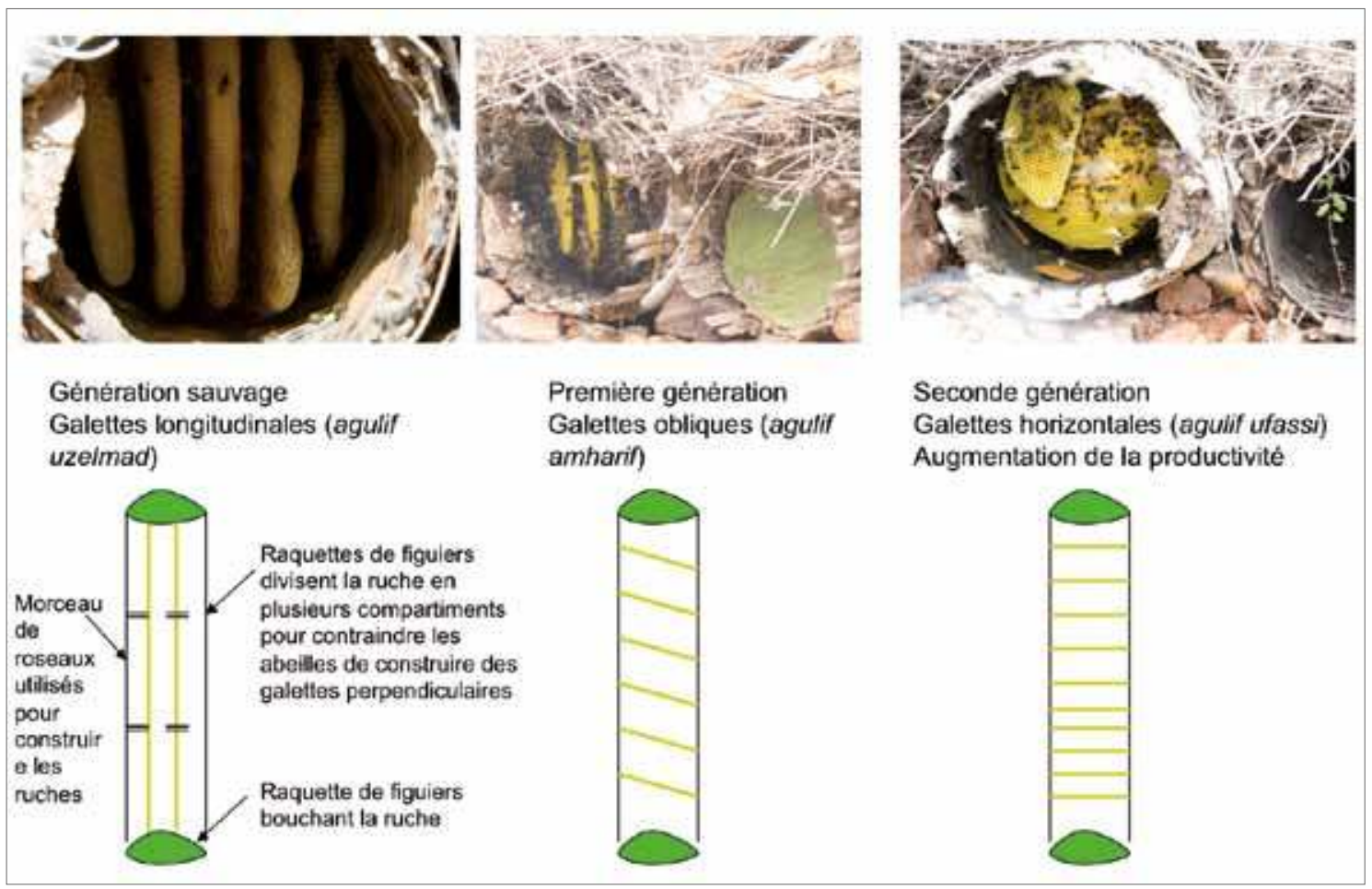


Pour déterminer à qui appartient un essaim accroché aux branches d'un arbre, l'usage est de le cendrer afin de repérer le soir venu auprès de l'entrée des ruches les quelques abeilles ainsi marquées qui ont souvent tendance à revenir vers leur lieu d'origine. Le territoire s'unifie ainsi lors de la période d'essaimage à la mesure du vol des essaims. Face à une telle mobilité des abeilles entre la gauche et la droite, la distinction entre ces deux catégories, tout aussi symbolique soit-elle, pourrait paraître obsolète. C'est sans compter l'ingéniosité technique des apiculteurs à l'origine d'une différence radicale dans l'architecture de l'habitacle entre celui des essaims de la gauche (agulif azelmad) et celui des essaims de la droite (agulif ufassi), l'un et l'autre se distinguant par une orientation différente des galettes. En effet, lorsqu'un nouvel essaim est cueilli par un apiculteur, qu'il soit prélevé sur des colonies en forêt ou bien issue de l'essaimage d'une ruche, il a tendance à fabriquer ses galettes dans la longueur de l'habitacle, de manière perpendiculaire à la sortie. Cette distinction ne sert pas seulement à opposer la structure de l'habitacle des essaims sauvages à celle de l'habitacle des essaims travaillés par l'homme, elle témoigne aussi de la transformation de l'habitacle d'une même ruche. Constituées, dans cette aire géographique, d'un clayonnage de roseaux fendus et en forme de tube, ces ruches sont souvent enduites d'un mélange de terre et d'excréments de vaches et fermées par des raquettes de figuiers de barbarie. Pour contraindre les abeilles à construire leurs galettes de manière parallèle à la sortie de l'habitacle, en épousant les rainures des roseaux d'armature, les apiculteurs introduisent dans la ruche des obstacles, souvent des raquettes de figuiers de barbarie, pour la diviser en plusieurs compartiments parallèles. Une telle architecture de la ruche permet notamment de faciliter l'extraction des galettes à miel, et apporte probablement des avantages aux colonies. Tant que les abeilles construisent leurs galettes de manière perpendiculaire, elles garderont leur statut d'abeilles de la gauche (uzelmad), ou d'abeilles sourdes (uderdur), et cela alors qu'elles se trouvent dans un rucher domestique. C'est bien l'intervention technique de l'apiculteur qui fait passer le plus rapidement possible l'abeille de la gauche vers la droite, rétablissant ainsi l'ordre du monde reposant sur la division entre espace domestique et espace sylvestre.

D'un point de vue purement technique, deux éléments peuvent expliquer cette manipulation, qui, selon les termes des manuels de formation apicole, correspond au passage d'une construction en bâtisse froide (rayons perpendiculaires à la sortie de la ruche) à une construction en bâtisse chaude (rayons parallèles à la sortie de la ruche). Tout d'abord, d'un point de vue pratique, les apiculteurs expliquent que la récolte se fait plus aisément par découpage des rayons entiers, rayons alors devenus visibles par l'apiculteur. Ensuite, on peut suggérer que cette opération vise un changement comportemental des abeilles, allant ainsi vers une moindre agressivité de la colonie, trait toujours apprécié par les apiculteurs en général. En effet, les abeilles d'une ruche aux rayons longitudinaux vont pouvoir facilement, d'un côté à l'autre de la ruche, se retrouver à l'extérieur, prêtes à l'attaque à la moindre secousse, ce qui n'est pas le cas lorsque le premier rayon obstrue le reste de la ruche. Ces deux éléments techniques consolident encore cette idée d'éducation de l'abeille: on lui « aménage » un espace et elle nous « donne » ses galettes plus facilement et plus docilement.

En forçant les abeilles à changer les structures de leur habitacle, les apiculteurs les incitent à créer un milieu différent de celui où elles se trouvent à l'état sauvage et scellent la distinction analogique entre « celles de la gauche » et « celles de la droite ». De ce fait, le sens d'« élever » et d'« éduquer » peut être littéralement appliqué à l'intervention des apiculteurs marocains sur les essaims puisque les abeilles sont entretenues dans une situation différente de celle où elles se trouvent à l'état sauvage (Tétart, 2001 : 181). Dans ce cas, les 
représentations symboliques ancrent les pratiques apicoles dans une polarité symbolique du territoire, entre la gauche et la droite, et, ce faisant, imposent l'aménagement du milieu comme cadre de la domestication de l'abeille.

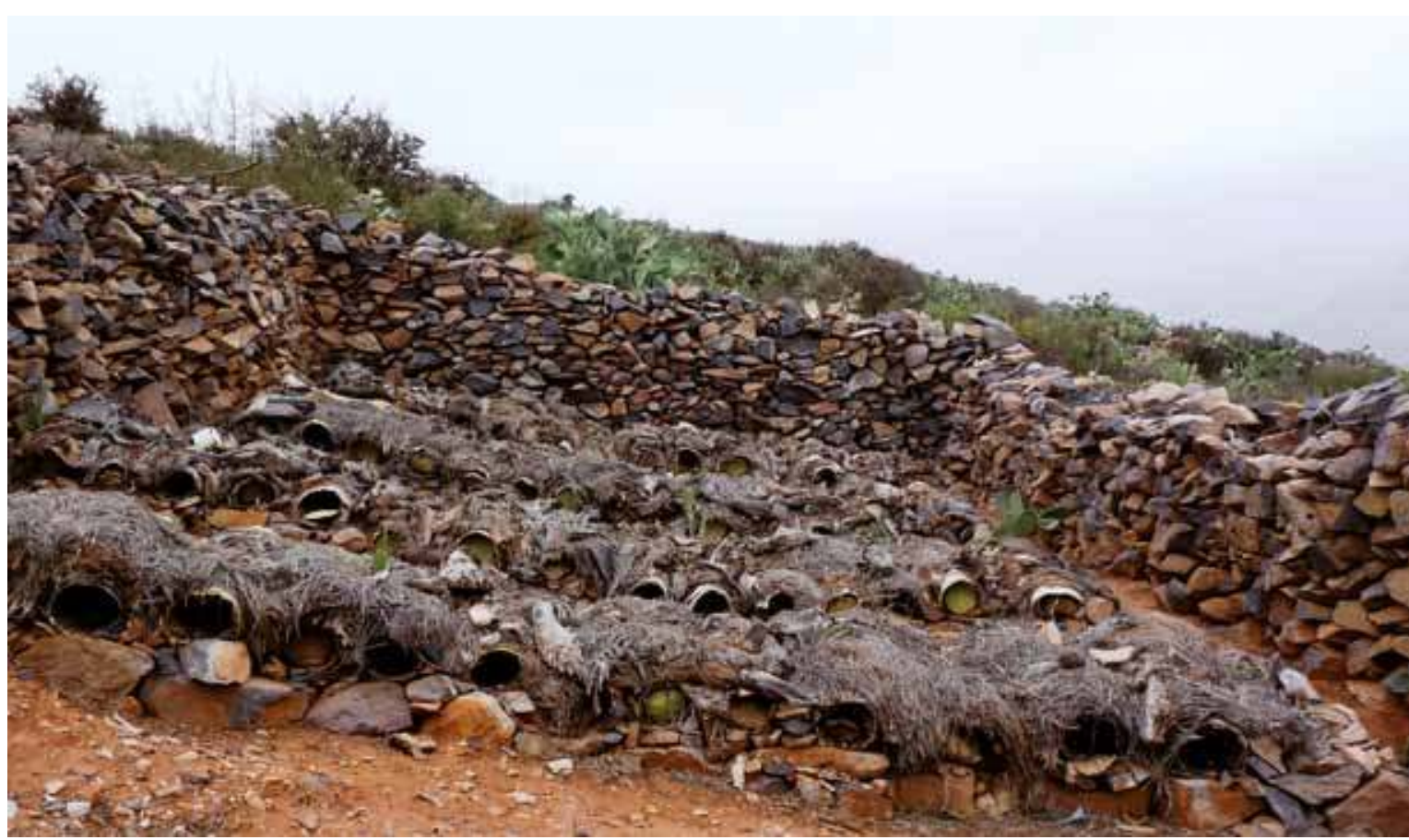

Souvent constitué d'un muret recouvert d'épineux, adossé au relief, et planté de figuiers de barbarie, il a pour vocation de protéger des agressions extérieures les colonies contre leur

\section{Une apiculture sédentaire holiste au sein du territoire rural}

Dans les régions semi-montagneuses du Sud atlantique Marocain, l'apiculture traditionnelle est réalisée au sein des territoires villageois. L'efficience de cette apiculture familiale à grande envergure repose sur trois critères, l'emplacement du site, la diversité des espaces de culture et des substances apicoles qu'ils abritent et l'étendue de la pollinisation.

\section{Le taddart}

Comme dans l'élevage caprin pratiqué dans ces régions du Sud marocain, les ruches et leurs abeilles sont «parquées » dans une sorte d'enclos. La comparaison avec le pastoralisme est inscrite dans la nomenclature apicole puisque le rucher enclos des abeilles porte un nom bien spécifique dans le langage tachelhit qui correspond au féminin de « bergerie »: taddart ou tagrourt.

Selon la structure des unités spatiales, le positionnement des taddart suit une logique d'optimisation précise au regard des connaissances, contraintes et exigences de l'apiculteur relatives à la production de miel, à l'accès aux matières apicoles, à l'ensoleillement, aux dérangement, leur destruction voire même leur pillage. 


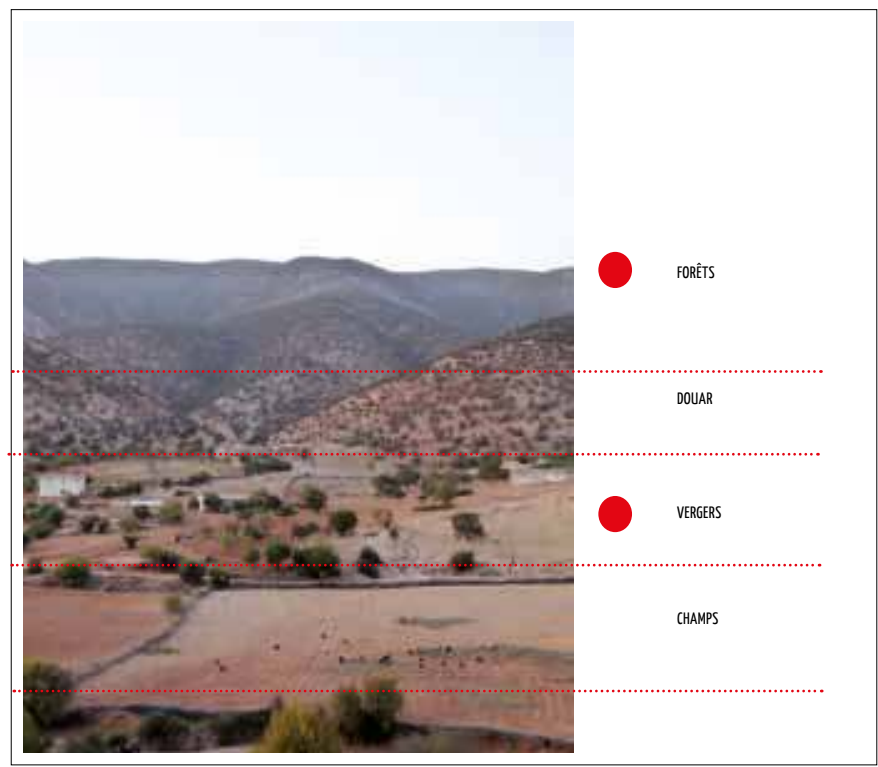

EMPLACEMENT POTENTIEL DES ABELLLES

Ce type de rucher accolé aux maisons peut prendre des proportions importantes dans certaines régions, comme dans celle d'Ida-0u-Tanane,

où les taddart comprennent une superposition de casiers en terre et en bois (tizghatine) contenant une ruche (ssild) et un espace supplémentaire pour l'extension de la colonie

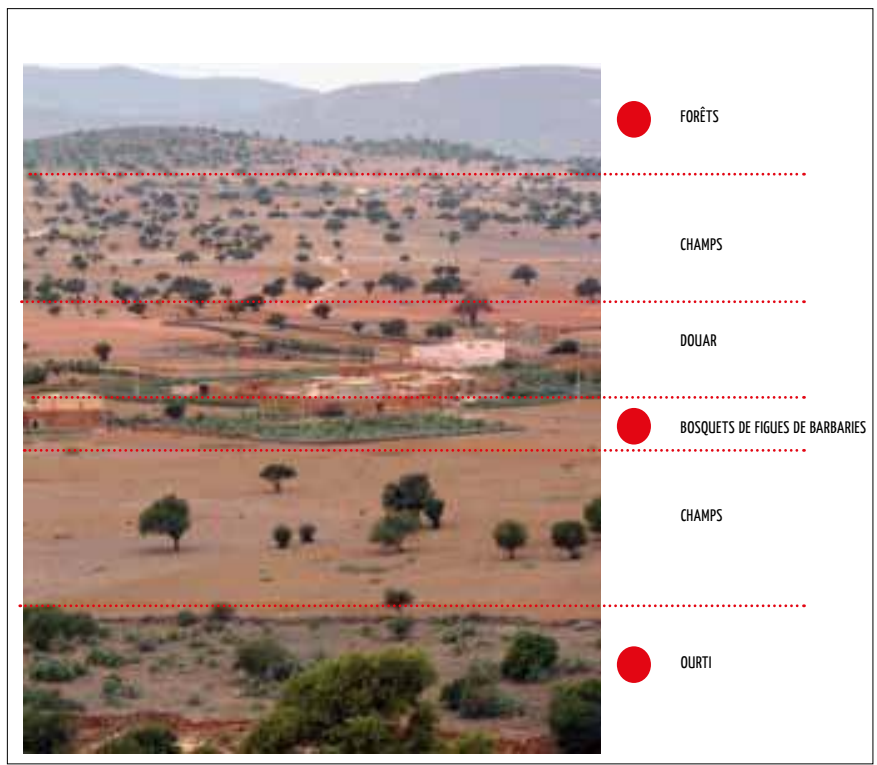

EMPLACEMENT POTENTIEL DES ABELLLES

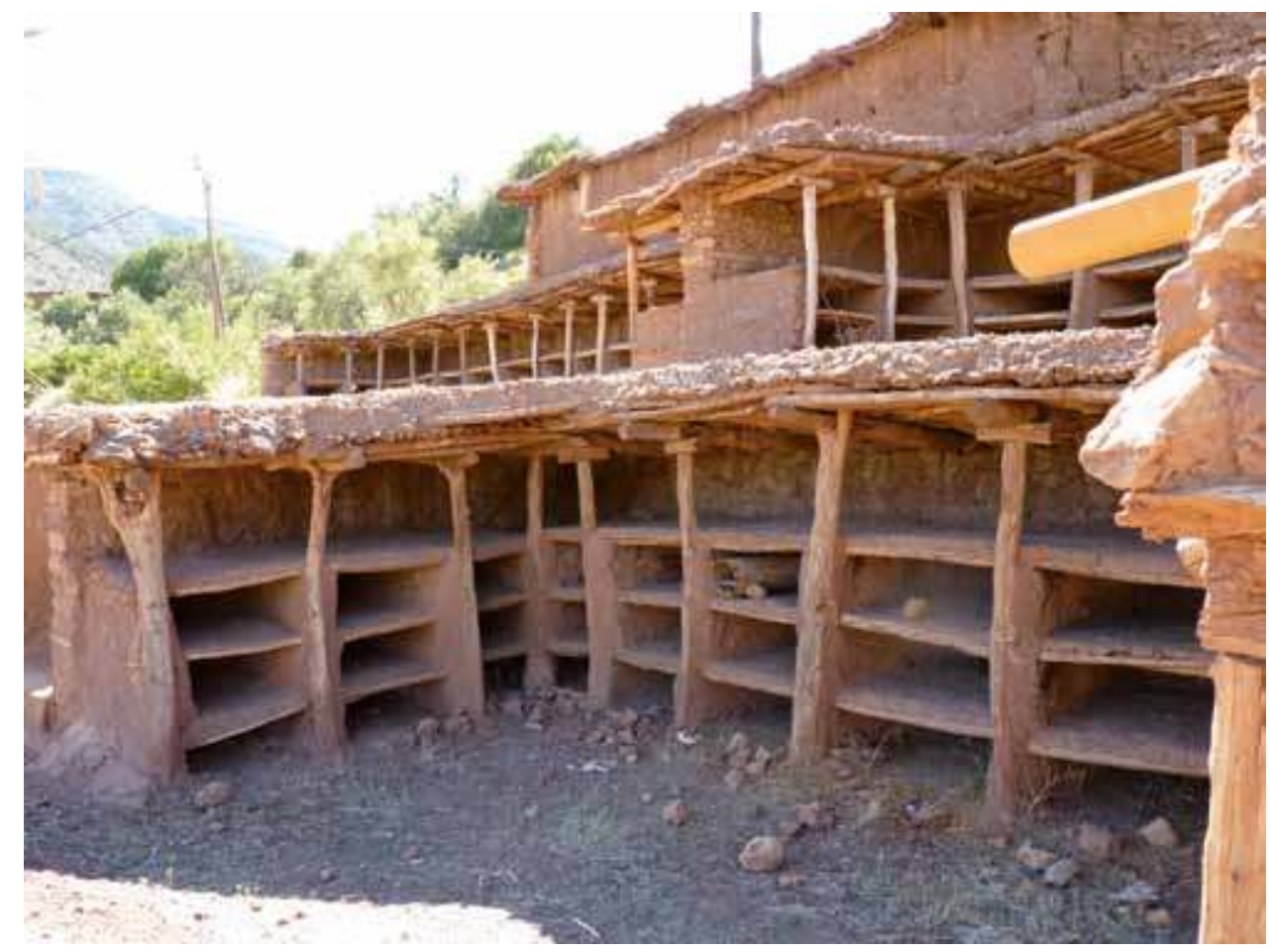


vents, à la facilité pour la récolte des essaims, à la pollinisation... Le taddart est avant tout le pivot d'une transhumance saisonnière entre les différents espaces cultivés (champs, jardins, ourti à figuiers de barbarie...) et forestiers d'un même territoire villageois.

Les taddart prennent trois formes différentes en fonction du positionnement dans le territoire des habitats humains.

Taddart de maison

Taddart des espaces cultivés

Taddart saisonnier ${ }^{5}$ dans des sites d'intérêt floristique particulier

Dans des territoires où les habitats sont positionnés aux croisements des espaces cultivés et forestiers, comme dans les vallées, les taddart sont le plus souvent juxtaposés aux maisons. La dimension du taddart dans des territoires où les habitats ne sont pas positionnés aux croisements des espaces de culture et sylvestres, comme en plaine, les taddart sont installés à des endroits stratégiques, dans des espaces intermédiaires entre terres cultivées et forêt. Lorsque les différentes unités spatiales agraires sont éloignées les unes des autres, ce qui est souvent le cas, une des solutions adoptées par les apiculteurs reste la construction de plusieurs taddart dans chacune de ces unités spatiales différentes. L'apiculteur pourra alors posséder plusieurs de ces ruchers afin d'avoir le choix de positionner plus précisément ses ruches selon ses besoins et/ou ceux de ses abeilles. Au grès des saisons et des années, celui-ci va déplacer ses ruches afin de combler au déficit d'un environnement local trop peu diversifié et/ou trop peu productif, au point de menacer la survie même des abeilles. Ainsi, le choix des zones plus humides des oasis lors de sécheresses, des zones arboricoles (ourti) pour la production de certains miels, des champs lors de l'essaimage, témoigne de l'adaptabilité éprouvée de l'apiculteur à un aléa climatique familier.

Une autre forme d'itinérance, qui s'approche plus d'une transhumance vers des zones inhabitées où est présente une flore spécifique, répond à une toute autre finalité: la production d'un miel particulier. L'exemple du thym illustre au mieux ce type de floraison, très prisée pour son miel. Dans ce cas, l'apiculteur va construire un taddart au cœur de l'aire de floraison. Il ne sera d'ailleurs souvent pas le seul à faire ce choix et ces espaces peuvent alors rassembler une multitude de taddart. L'habitude de transformer certaines aires de floraison en véritables parcours collectifs pour les abeilles est probablement à l'origine de l'institution de ruchers collectifs dans ces régions du Sud marocain. Ces ruchers collectifs sont bien évidemment soumis à des règles coutumières d'accès à la ressource.

Aujourd'hui, l'utilisation de ruches « modernes », types Langstroth ou Dadant, par certains apiculteurs, tend à modifier ces positions stratégiques, notamment par la possibilité et surtout la nécessité de transhumer ce type de ruches ${ }^{6}$. Néanmoins, les situations sont diverses en fonction des stratégies choisies par ces apiculteurs: certains cas d'apiculture moderne empruntent ou conservent la démarche de la position holiste de l'apiculture traditionnelle au sein du système agraire. À titre d'exemple, certains apiculteurs des lieux où l'habitat est condensé au centre des espaces de culture et qui adossaient les ruches contre leur maison transhument aujourd'hui les ruches modernes sur le toit de cette même habitation. Autant que possible, les apiculteurs qui changent leurs pratiques conservent les emplacements de leurs anciens taddart pour mettre leurs nouvelles ruches, tout en y préservant souvent quelques ruches traditionnelles fixes lorsqu'ils transhument le reste. Les uns disent que c'est pour maintenir la production d'un miel local, d'autres affirment assurer ainsi une sorte de gardiennage de leur emplacement, quoi qu'il en soit, une telle attitude témoigne d'une certaine pertinence locale de l'apiculture comme un élément de l'équilibre écologique et sociologique 


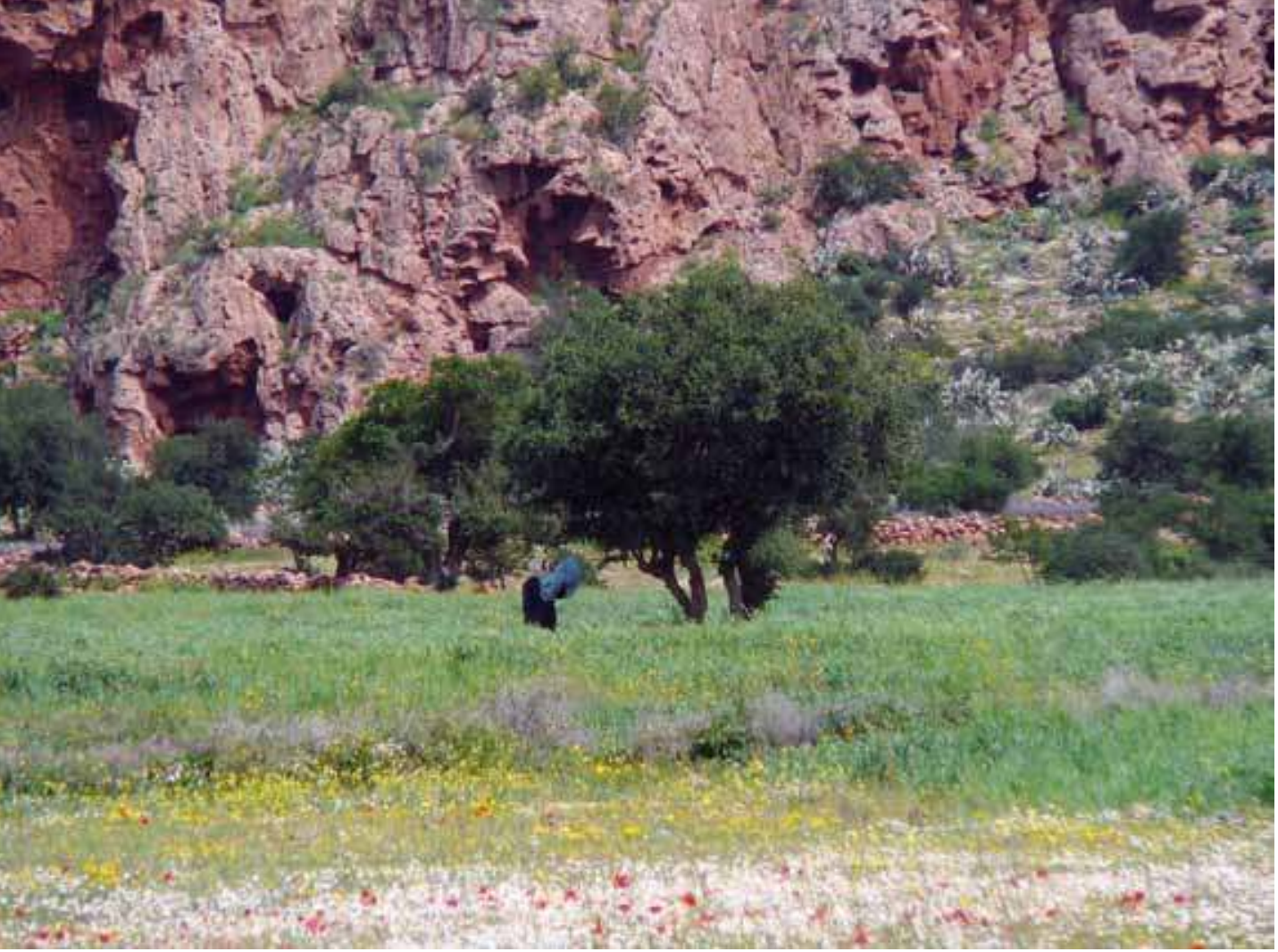

Falaise : Habitat des essaims sauvages

Dans les ruchers collectifs, toujours positionnés dans des sites stratégiques du point de vue du couvert floristique, les apiculteurs de tribus différentes placent leurs ruches sous la surveillance de gardiens de ces territoires ruraux. L'apiculture dans ce cas, même si elle va occuper une place plus importante dans le calendrier de travail de ces nouveaux apiculteurs, reste une activité intégrée au système agraire. L'élevage et les cultures sont toujours pratiqués, les relations existantes entre ces différentes composantes agraires formant l'agrosystème perdurent et renforcent encore l'idée d'une agriculture très étroitement liée à l'apiculture, et ce à de nombreux niveaux.

\section{b) À chaque espace de culture, une dimension technique de la domestication de labbeille}

Le territoire est constitué d'une diversité d'unités spatiales complémentaires qui supportent des dimensions diverses de la domestication des abeilles.

1. Falaises et cascades: souvent présentes en marge du territoire villageois ou tribal, elles représentent l'habitat naturel privilégié des abeilles sahariennes. La capture d'essaim sauvage est, après une période de sécheresse ou pour débuter son activité, une activité essentielle pour l'apiculture traditionnelle du sud marocain. De nombreuses techniques existent pour capturer l'essaim qui s'installe principalement dans les zones de falaises et parfois en forêt. Le plus simple, mais aussi le plus rare, si l'essaim est visible et accessible facilement, reste d'amener un panier et d'y secouer l'essaim. Cette opération est autrement plus délicate si elle se déroule à plusieurs mètres au-dessus du sol. Une autre technique qui doit être préparée très à l'avance consiste en la construction d'une cavité au sol dont on peut ôter le couvercle (pierre plate, planche...). La cavité sert en quelque sorte d'habitacle appât afin d'attirer l'essaim. Une fois que l'essaim s'y est installé et qu'il y a bâti ses premiers rayons, l'apiculteur procède au transvasement dans une ruche, qu'il emmènera le soir venu jusqu'au taddart. Ces essaims peuvent être soit récupérés, soit récoltés, soit les deux. Certains apiculteurs sont spécialisés dans ces activités de manière à vendre miel et essaims tout au long d'un printemps généreux.

2. Des grands champs entourent de petites zones de hameaux qui sont généralement encerclées par des zones de culture de figuiers de barbarie, véritable barrière épineuse protégeant à la fois les hommes et le bétail autour de la maison. En bordure de l'espace habité, ces bosquets de figuiers de barbarie servent pour les apiculteurs d'espace de multiplication des essaims. Lors de l'essaimage, les essaims se dirigent naturellement vers les arganiers 
des champs, ce qui rend leur localisation et leur capture beaucoup plus aisée que dans la forêt.

3. Les champs sont de vastes zones de culture de céréales (orge et blé) recouvertes d'arganiers dispersés, d'amandiers ou encore de caroubiers. Les arganiers et autres arbres mellifères trônant dans les champs fournissent un abri pour les nouveaux essaims d'abeilles, qu'ils proviennent des falaises ou de l'essaimage des ruches domestiques.

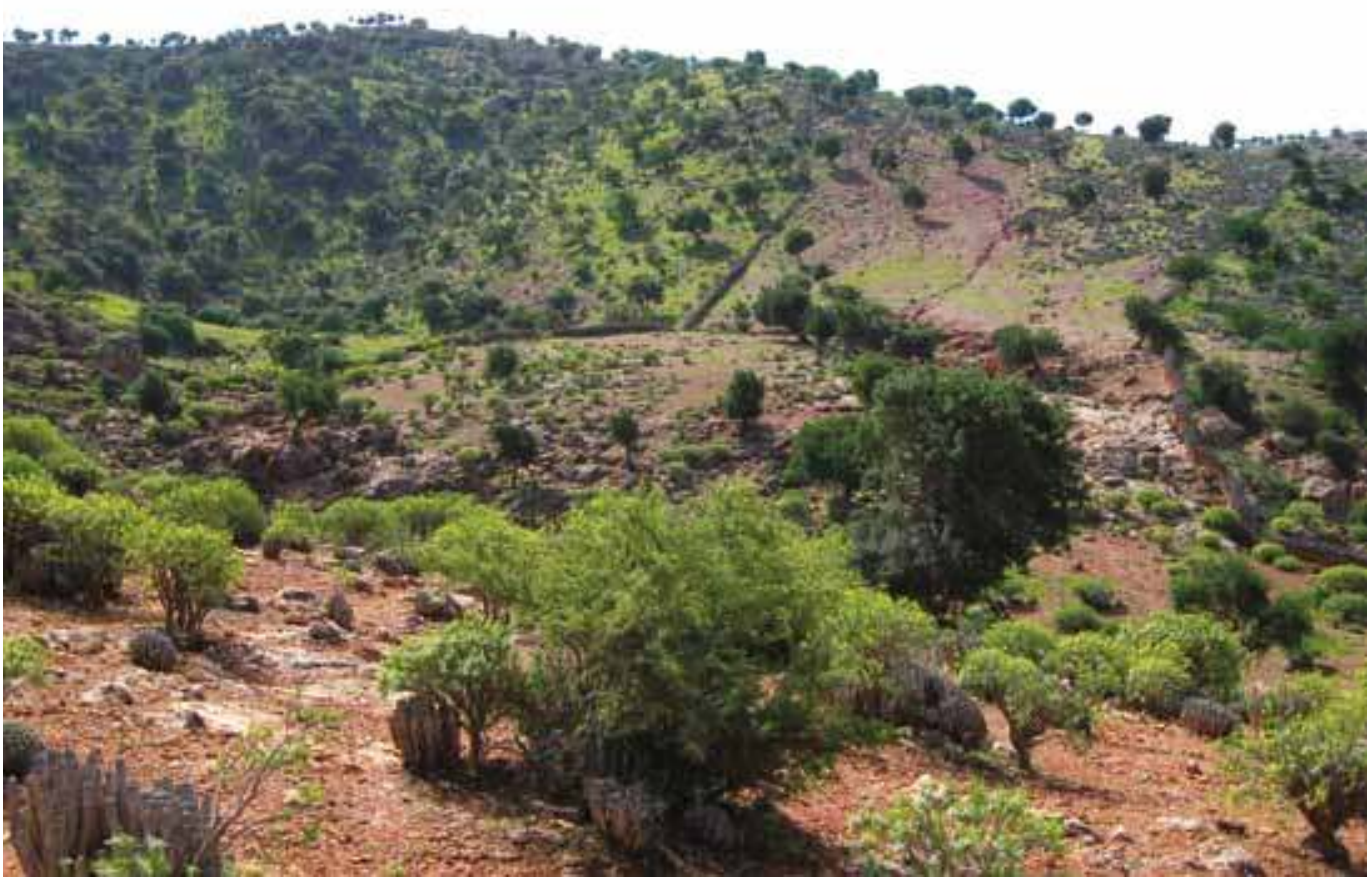
Le stock de nectar de Espace arboricole (ourti) : production de miel fleurs d'arganiers est suffisant pour les attirer le temps de les acclimater aux ruches avant de pouvoir être déplacés. Ces arbres isolés dans le paysage agraire constituent une première étape de l'acclimatation des abeilles dans un paysage fortement domestiqué que sont les champs. À la fin du printemps, les arganiers champêtres sont ainsi truffés d'essaims en forme de boules. Parfois, les apiculteurs installent de vieilles ruches à l'odeur de miel dans les jujubiers ou les arganiers des champs pour attirer les nouveaux essaims à peine sortis de leurs habitats d'origine, facilitant ainsi leur transvasement.

4. À l'extérieur des plaines agricoles, sur les pentes montagneuses, s'étendent des forêts clairsemées d'arganiers qui servent de zones de pacage pour les chèvres ainsi que de récolte pour les noix d'argan (Simenel \& al., 2009). La densité des arganiers permet le développement d'un couvert floristique très diversifié en partie méditerranéen, en partie océanien, en partie saharien. Les forêts d'arganiers abritent ainsi tout un stock de nectar et autres matières apicoles comme la propolis. La cohabitation de certaines espèces végétales comme celle entre l'euphorbe cactoïde et le Senecio anteuphorbium L., deux espèces de floraison estivale, offre aux abeilles le panel de matières apicoles nécessaire à la fabrication d'un des miels les plus prisés du Maghreb.

5. Entre les champs et la forêt s'interposent de-ci de-là des ourti, des zones arboricoles closes disposant d'infrastructures d'aménagement du sol et contenant des arganiers entretenus et des espèces cultivées telles que le henné et le figuier de barbarie. Attenants à la forêt, ces ourti constituent le domaine de prédilection des apiculteurs pour y entreposer leurs ruches dans l'optique de produire du miel.

6. Au pied des falaises, se trouvent parfois de petites oasis aménagées en jardins irrigués où sont cultivés des arbres fruitiers. Ces petites oasis constituent pour l'apiculture des zones spécifiques où l'eau est disponible toute l'année principalement en raison de la proximité des cours d'eau et/ou de la présence d'une source. En période de sécheresse 
prolongée, qui s'étend sur plus de deux années successives, les apiculteurs ont l'habitude d'y réfugier leurs abeilles afin qu'elles y puisent l'eau nécessaire pour survivre.

Suivant les années et ses priorités, l'apiculteur décidera ainsi de positionner ses ruches plutôt dans un ourti, s'il cherche à produire du miel, dans un espace de figuiers de barbarie en lisière des hameaux, s'il cherche à multiplier ses essaims rapidement au printemps, ou dans les petits jardins oasis pour sauvegarder ses abeilles en cas de sécheresse prolongée.

\section{c) De l'emplacement des ruches à la pollinisation des arbres}

Le choix de l'emplacement des ruches dans une telle mosaïque de territoires cultivés n'est pas anodin. Maintenir les ruches à proximité des maisons dans les figuiers de barbarie ou les installer dans une zone arboricole fermée en lisière des champs incite les abeilles à la pollinisation des arganiers situés en zone agricole. Partant de ces deux emplacements, les abeilles peuvent ainsi parcourir une distance allant des habitations aux forêts d'arganiers, couvrant ainsi l'ensemble de l'espace cultivé. Le besoin en pollinisation est d'autant plus important dans les champs que le nombre d'arganiers fructifiant deux fois dans l'année y est conséquent, contrairement aux espaces forestiers. De plus, amener les abeilles à butiner les fleurs d'arganiers des champs permet, selon les apiculteurs, de doubler la production de fruits. Ainsi les arganiers des champs donnent la majorité de la récolte en fruits, matière première de l'huile d'argan. L'objectif ici est autant la pollinisation des arbres des champs que la mise à disposition d'une grande variété de matières apicoles pour les abeilles, puisque de nombreuses plantes messicoles en fournissent. La pollinisation des arganiers de forêts est assurée par les essaims domestiques des ourti, et sauvages, logés dans les falaises, et par les autres insectes ou mammifères pollinisateurs. Quant aux petites oasis, les arbres fruitiers sont pollinisés par les abeilles sauvages, ou par les abeilles domestiques qui y sont entreposées en période de sécheresse.

La maîtrise du vol des abeilles est à ce point considéré comme un critère de leur domestication que, lorsque l'apiculteur Ait Ba'amran décrit leurs trajectoires, il les compare à un troupeau qui sort du village, traverse les champs en pâturant ce qu'il y a de bon à pâturer, pour finir par se frayer un chemin vers la forêt avant de revenir le soir à la maison.

\section{Temporalité des ressources apicoles et connaissances sur le métabolisme des abeilles}

D'un point de vue temporel, aux différentes saisons coïncide une disponibilité en pollens et nectars provenant d'espèces de fleurs variées réparties dans la diversité des unités spatiales composant le territoire rural. Cette complémentarité permet de produire, tout au long de l'année, différents types de miel, excepté en période de sécheresse prolongée.

$1 .{ }^{7}$ De décembre à mars, les euphorbes balsamiques ${ }^{8}$ présentes dans les ourti et les forêts sont en fleurs, et leur nectar donne un miel particulièrement sucré, réputé pour ses vertus digestives. Si l'année ne s'annonce pas assez pluvieuse, les apiculteurs laissent cette première récolte aux abeilles.

2. De mars à mai, les fleurs d'espèces adventices sont disponibles dans les champs de céréales comme celles de certains asphodèles, de liserons ainsi que les fleurs d'arganiers dans les champs, les forêts et les ourti. Là encore, selon la pluviométrie de l'année, la part de miel ponctionnée par l'apiculteur varie. 


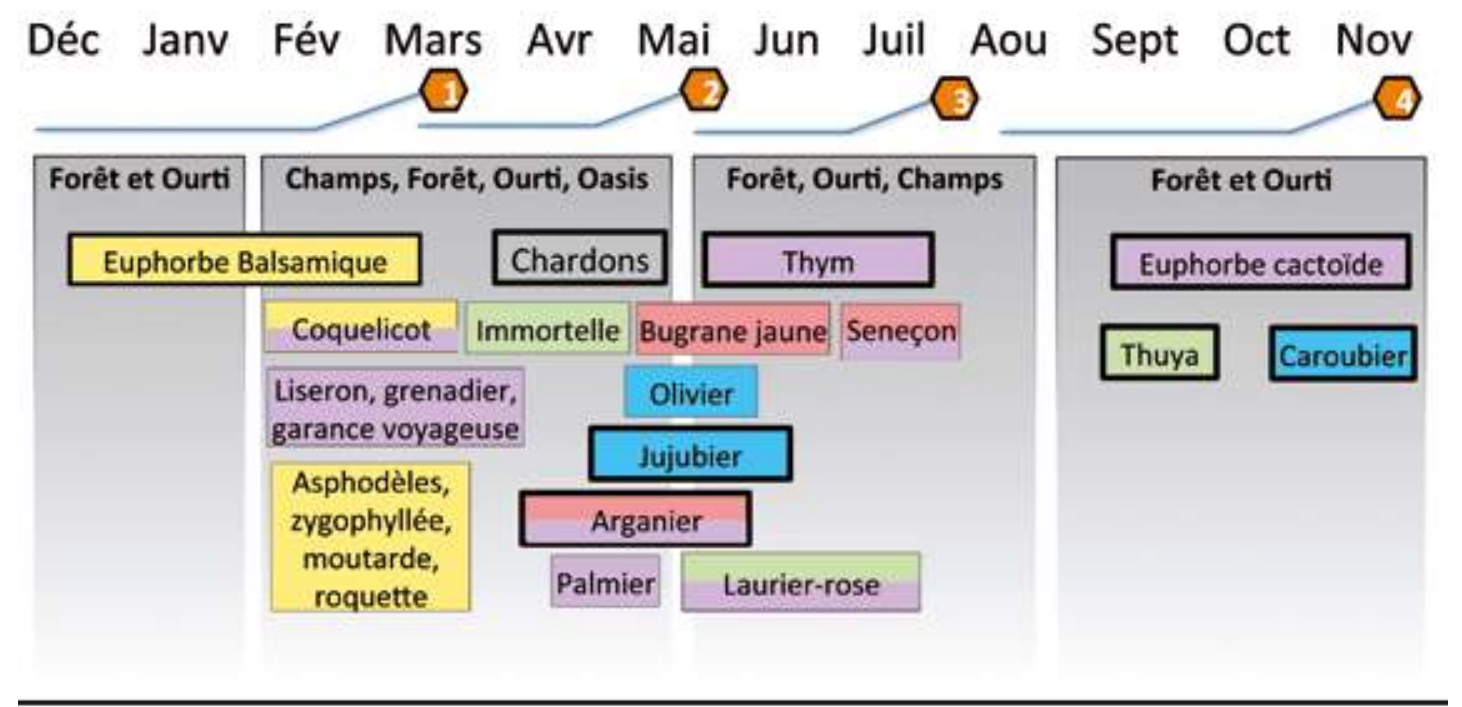

\section{Légende:}

\begin{tabular}{|l|}
\hline Augmentation fréquence d'essaimage \\
\hline Diminution de la fréquence d'essaimage \\
\hline Santé et hygiène de la ruche \\
\hline Santé et hygiène de la ruche: source de propolis \\
\hline Diminution de la capacité de travail des abeilles \\
\hline
\end{tabular}

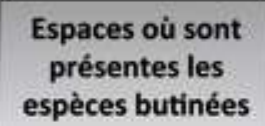

Saisons de miellée: -

Récolte possible: $\square$

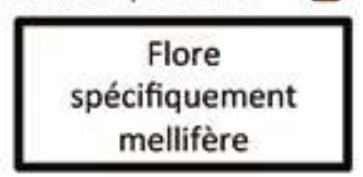

3. De mai à juin, un autre ensemble d'espèces adventices envahit les champs ouverts, comme le chardon ou le jujubier, dont les fleurs sont abondamment mellifères. Le miel de chardon ou de jujubier s'avèrent être deux des miels les plus réputés de ces régions après les miels d'euphorbe cactoïde et de thym. Ce dernier tient une place privilégiée pour ses propriétés thérapeutiques, et la floraison du thym s'étend principalement sur les mois de juin et juillet.

4. Enfin, entre juillet et novembre, ces fameuses euphorbes, endémiques de la zone, produisent un miel célèbre au Maroc pour ses vertus thérapeutiques.

Ainsi, les régions du Sud ouest marocain, toutes présahariennes qu'elles soient, offrent un panel de pollens et de nectars aux abeilles qui se déploie tout au long de l'année. Les abeilles de cette région, dites sahariennes (Apis mellifera sahariensis), ont évolué avec ce complexe floristique et dans un climat particulier auquel elles se sont adaptées pouvant butiner l'été sous $45^{\circ} \mathrm{C}$ à l'ombre. Excepté en cas de sécheresse, il n'y a pas de période de soudure, celles-ci ont en effet de quoi s'alimenter et produire du miel toute l'année grâce à la diversité des types de territoires cultivés et des écosystèmes qu'ils abritent.

Mais les abeilles ne récoltent pas que du nectar et du pollen de toutes ces unités du territoire rural, elles rapportent aussi une gamme non négligeable de matières apicoles indispensables à la bonne hygiène et à la reproduction de l'essaim. Les apiculteurs interprètent tous les aspects du cycle de vie des abeilles en tenant compte des usages 


\begin{tabular}{|c|c|c|c|c|c|}
\hline$\frac{\text { Tachelhit }}{\text { Arabe }}$ & $\begin{array}{c}\text { Nom } \\
\text { français }\end{array}$ & $\begin{array}{c}\text { Nom } \\
\text { scientifique }\end{array}$ & Substance & $\begin{array}{l}\text { Localisation } \\
\text { écologique }\end{array}$ & $\begin{array}{c}\text { Connaissance } \\
\text { région }^{1}\end{array}$ \\
\hline Tallalt & $\begin{array}{c}\text { Euphorbe } \\
\text { balsamique ou } \\
\text { arborescente }\end{array}$ & $\begin{array}{c}\text { Euphorbia } \\
\text { balsamifera } \\
\text { Aiton } \\
\text { Euphorbia } \\
\text { obtusifolia } \\
\text { Poir. }\end{array}$ & Pollen/Nectar & $\begin{array}{l}\text { Forêt, } \\
\text { Ourti }\end{array}$ & Individuelle \\
\hline 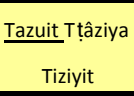 & $\begin{array}{c}\text { Petite } \\
\text { asphodèle }\end{array}$ & $\begin{array}{c}\text { Asphodelus } \\
\text { tenuifolius } \\
\text { Cav. }\end{array}$ & Pollen/Nectar & Champs & $\begin{array}{l}\text { Partagée } \\
\text { MT/SMD }\end{array}$ \\
\hline $\begin{array}{l}\text { Igri/Ingri } \\
\text { I-berwâg }\end{array}$ & $\begin{array}{l}\text { Asphodèle à } \\
\text { petits fruits }\end{array}$ & $\begin{array}{c}\text { Asphodelus } \\
\text { microcarpus } \\
\text { Viv. }\end{array}$ & Pollen/Nectar & Champs & $\begin{array}{l}\text { Partagée } \\
\text { GS/SMD }\end{array}$ \\
\hline Kerkâz & $\begin{array}{c}\text { Diplotaxes } \\
\text { Moutardes } \\
\text { Faux rapistre }\end{array}$ & $\begin{array}{c}\text { Diplotaxis sp. } \\
\text { Brassica sp. } \\
\text { Hirschfeldia } \\
\text { sp. } \\
\end{array}$ & Pollen/Nectar & Champs & $\begin{array}{l}\text { Partagée } \\
\text { MT/SMD }\end{array}$ \\
\hline Berber & $\begin{array}{l}\text { Roquette } \\
\text { bâtarde }\end{array}$ & $\begin{array}{c}\text { Hirschfeldia } \\
\text { incana (L.) } \\
\text { Lagr.-Foss }\end{array}$ & Pollen/Nectar & Champs & $\begin{array}{c}\text { Anecdotique } \\
\text { SMD }\end{array}$ \\
\hline I-'aggâya & Zygophylle & $\begin{array}{c}\text { Zygophyllum } \\
\text { sp. }\end{array}$ & Pollen/Nectar & Champs & $\begin{array}{c}\text { Individuelle } \\
\text { GS }\end{array}$ \\
\hline $\begin{array}{l}\text { Oussoussen } \\
\text { Bena'mân }\end{array}$ & Coquelicot & $\begin{array}{l}\text { Papaver } \\
\text { rhoeas } \mathrm{L} . \\
\text { Papaver } \\
\text { dubium } \mathrm{L} .\end{array}$ & Pollen/Nectar & Champs & $\begin{array}{l}\text { Partagée } \\
\text { MT/SMD }\end{array}$ \\
\hline$\frac{\text { Timerzgelid }}{\text { Azatîm }}$ & $\begin{array}{l}\text { Immortelle } \\
\text { bleue }\end{array}$ & $\begin{array}{c}\text { Limonium } \\
\text { sinuatum (L.) } \\
\text { Mill. }\end{array}$ & Pollen/Nectar & Champs & $\begin{array}{c}\text { Individuelle } \\
\text { GS }\end{array}$ \\
\hline $\begin{array}{l}\text { Azuka } \\
\text { 'ar'ar }\end{array}$ & Thuya & $\begin{array}{c}\text { Tetraclinis } \\
\text { articulata } \\
\text { (Vahl) Mast. }\end{array}$ & Miellat & Forêt & $\begin{array}{l}\text { Partagée } \\
\text { MT/SMD }\end{array}$ \\
\hline$\frac{\text { Alilî }}{\text { Ddeflâ }}$ & Laurier rose & $\begin{array}{c}\text { Nerium } \\
\text { oleander L. }\end{array}$ & Bois & Oued & $\begin{array}{c}\text { Individuelle } \\
\text { GS }\end{array}$ \\
\hline
\end{tabular}

\begin{tabular}{|c|c|c|c|c|c|}
\hline$\frac{\text { Tachelhit }}{\text { Arabe }}$ & $\begin{array}{c}\text { Nom } \\
\text { français }\end{array}$ & $\begin{array}{c}\text { Nom } \\
\text { scientifique }\end{array}$ & Substance & $\begin{array}{l}\text { Localisation } \\
\text { écologique }\end{array}$ & $\begin{array}{c}\text { Connaissance } \\
\text { région }^{1}\end{array}$ \\
\hline $\begin{array}{c}\text { Aselift } \\
\text { El- ḥallâb }\end{array}$ & Pas de nom & $\begin{array}{c}\text { Periploca } \\
\text { laevigata } \\
\text { Aiton }\end{array}$ & $\begin{array}{c}\text { Piquante } \\
\text { Latex } \\
\end{array}$ & Forêt & $\begin{array}{l}\text { Partagée } \\
\text { MT/GS }\end{array}$ \\
\hline Argane & Arganier & $\begin{array}{l}\text { Argania } \\
\text { spinosa L. }\end{array}$ & Lait/Latex & $\begin{array}{c}\text { Forêt } \\
\text { Ourti } \\
\text { Champs }\end{array}$ & $\begin{array}{l}\text { Partagée } \\
\text { MT/SMD }\end{array}$ \\
\hline $\begin{array}{l}\text { Achbarto } \\
\text { Sbârto }\end{array}$ & Seneçon & $\begin{array}{c}\text { Senecio } \\
\text { anteuphorbiu } \\
m \mathrm{~L} .\end{array}$ & Pollen/Nectar & $\begin{array}{c}\text { Forêt } \\
\text { (association } \\
\text { avec Tkiût) }\end{array}$ & $\begin{array}{l}\text { Partagée } \\
\text { GS/SMD }\end{array}$ \\
\hline $\begin{array}{l}\text { Afesdad } \\
\text { Afzzaz } \\
\text { Fezzaz }\end{array}$ & $\begin{array}{c}\text { Bugrane jaune } \\
\text { Ononis de } \\
\text { tournefort }\end{array}$ & $\begin{array}{c}\text { Ononis natrix } \\
\text { L. } \\
\text { Ononis } \\
\text { tournefortii } \\
\text { Coss. }\end{array}$ & $\begin{array}{l}\text { Exsudat } \\
\text { collant à la } \\
\text { surface de } \\
\text { toute la } \\
\text { plante }\end{array}$ & $\begin{array}{c}\text { Forêt } \\
\text { Champs }\end{array}$ & $\begin{array}{l}\text { Partagée } \\
\text { SMD }\end{array}$ \\
\hline $\begin{array}{c}\frac{\text { Tumzîn }}{\text { Ě̌-š'îr }} \\
\text { Zra' }\end{array}$ & Orge & $\begin{array}{l}\text { Hordeum } \\
\text { vulgare } \mathrm{L} .\end{array}$ & $\begin{array}{c}\text { Exsudat à } \\
\text { l'insertion de } \\
\text { la feuille }\end{array}$ & Champs & $\begin{array}{c}\text { Individuelle } \\
\text { MT }\end{array}$ \\
\hline$\frac{\text { Azar }}{\text { Kerma }}$ & Figuier & Ficus carica L. & Latex & Jardins & $\begin{array}{l}\text { Partagée } \\
\text { GS/SMD }\end{array}$ \\
\hline Zaytûn & Olivier & $\begin{array}{c}\text { Olea } \\
\text { europaea L. }\end{array}$ & Pollen/Nectar & $\begin{array}{l}\text { Jardins } \\
\text { Champs }\end{array}$ & $\begin{array}{c}\text { Individuelle } \\
\text { MT }\end{array}$ \\
\hline $\begin{array}{c}\text { Azuggwar } \\
\text { Ssder } \\
\text { Sedra }\end{array}$ & Jujubier & $\begin{array}{l}\text { Zizyphus lotus } \\
\text { (L.) Lam. }\end{array}$ & Epines & Champs & $\begin{array}{c}\text { Individuelle } \\
\text { SMD }\end{array}$ \\
\hline$\frac{\text { Tikida }}{\text { I-ḥerrôb }}$ & Caroubier & $\begin{array}{l}\text { Ceratonia } \\
\text { siliqua } \mathrm{L} .\end{array}$ & Sève & Forêt & $\begin{array}{c}\text { Anecdotique } \\
\text { SMD }\end{array}$ \\
\hline$\frac{\text { Tazdâyt }}{\text { Nehla }}$ & Palmier mâle & $\begin{array}{c}\text { Phoenix } \\
\text { dactylifera L. }\end{array}$ & Pollen & $\begin{array}{l}\text { Oasis } \\
\text { Oued }\end{array}$ & $\begin{array}{c}\text { Individuelle } \\
\text { GS }\end{array}$ \\
\hline Azar & Figuier & Ficus carica L. & Eau & Jardins & Individuelle \\
\hline $\begin{array}{l}\frac{\text { Tansfelt }}{\text { Luwâya }} \\
\text { Luwayg }\end{array}$ & $\begin{array}{l}\text { Le liseron } \\
\text { fausse- } \\
\text { guimauve }\end{array}$ & $\begin{array}{l}\text { Convolvulus } \\
\text { althaeoides L. }\end{array}$ & Pollen & Champs & $\begin{array}{l}\text { Partagée } \\
\text { MT/SMD }\end{array}$ \\
\hline $\begin{array}{l}\text { Tarommant } \\
\text { Er-rummân }\end{array}$ & Grenadier & $\begin{array}{c}\text { Punica } \\
\text { granatum L. }\end{array}$ & Eau dans fleur & Jardins & $\begin{array}{c}\text { Individuelle } \\
\text { GS } \\
\end{array}$ \\
\hline$\frac{\text { Tazuknit }}{\text { Za'atar }}$ & $\begin{array}{c}\text { Thyms } \\
\text { et Sarriettes }\end{array}$ & $\begin{array}{l}\text { Thymus sp. } \\
\text { Satureja sp. }\end{array}$ & Toute la plante & Forêt & $\begin{array}{l}\text { Partagée } \\
\text { MT/SMD }\end{array}$ \\
\hline Ddag̉hmûs & $\begin{array}{l}\text { Euphorbe } \\
\text { cactoïde }\end{array}$ & \begin{tabular}{|c|} 
Euphorbia \\
officinarum \\
Hook.f. \& Coss. \\
(subsp. \\
officinarum et \\
subsp. echinus) \\
\end{tabular} & Latex & Forêt & $\begin{array}{l}\text { Partagée } \\
\text { GS/SMD }\end{array}$ \\
\hline$\frac{\text { Tzart n-îfiss }}{\text { Afessas }}$ & Pas de nom & $\begin{array}{c}\text { Warionia } \\
\text { saharae Benth. }\end{array}$ & Latex & Forêt & $\begin{array}{c}\text { Individuelle } \\
\text { GS }\end{array}$ \\
\hline $\begin{array}{l}\frac{\text { Izrî }}{\text { Sîh }} \\
\end{array}$ & Armoise & $\begin{array}{c}\text { Artemisia herba } \\
\text { alba Asso }\end{array}$ & $\begin{array}{l}\text { Toute la plante } \\
\text { sèche }\end{array}$ & $\begin{array}{c}\text { Forêt } \\
\text { Pâturages } \\
\text { d'altitudes }\end{array}$ & $\begin{array}{l}\text { Individuelle } \\
\text { SMD }\end{array}$ \\
\hline $\begin{array}{l}\text { Karbûn } \\
\text { Karbûr }\end{array}$ & $\begin{array}{c}\text { Carbure de } \\
\text { calcium }\end{array}$ & $\mathrm{CaC}_{2}$ & Minéral & $x$ & $\begin{array}{c}\text { Individuelle } \\
\text { SMD } \\
\end{array}$ \\
\hline$\frac{\text { Alilî }}{\text { Ddeflâ }}$ & Laurier rose & $\begin{array}{c}\text { Nerium } \\
\text { oleander L. }\end{array}$ & Eau des fleurs & Oued & $\begin{array}{c}\text { Individuelle } \\
\text { GS }\end{array}$ \\
\hline Tarûbiya Nsega & $\begin{array}{c}\text { Garance } \\
\text { voyageuse }\end{array}$ & $\begin{array}{c}\text { Rubia peregrina } \\
\text { L. }\end{array}$ & Colle & Champs & $\begin{array}{c}\text { Individuelle } \\
\text { GS }\end{array}$ \\
\hline
\end{tabular}


qu'elles font des substances végétales qu'elles récoltent. Ils en évaluent alors leurs effets sur le développement de la ruche et le comportement de ses habitantes. Que ce soit de manière naturelle ou provoquée, l'essaimage est une étape incontournable pour permettre le développement du rucher. Aussi, les apiculteurs ont relevé l'effet de certaines plantes sur cette étape. Par exemple, le petit asphodèle poussant dans les champs est reconnu pour son nectar stimulant la ponte de la reine, d'où son surnom de « viagra des abeilles ». Concernant l'hygiène, l'exsudat des feuilles de céréales, parmi d'autres substances, serait utilisé par les abeilles pour le nettoyage de la ruche, notamment des rainures des roseaux avec lesquels elle est fabriquée. La disponibilité de ce cortège végétal découle d'une concordance spatio-temporelle remarquable entre les pratiques humaines et l'activité des abeilles.

Sur la base des connaissances des apiculteurs, certaines plantes peuvent jouer un rôle essentiel dans le déroulement des différentes étapes constitutives de l'apiculture, de l'évolution du couvain à l'essaimage. Nous avons pu classer les différents effets et usages des substances végétales récoltées par les abeilles en cinq catégories (tableau 1):

- Augmentation de la fréquence d'essaimage par réduction du temps de latence. La récolte des substances de ces plantes par les abeilles aurait tendance à augmenter la fréquence d'essaimage en stimulant l'agressivité des abeilles. L'effet de la plante est d'abord constaté par les apiculteurs dans la manière dont les abeilles protègent plus férocement le couvain lors de la période de récolte de ces substances. Ensuite, les apiculteurs remarquent que le temps de latence entre chaque essaimage est raccourci lors de la floraison de ces espèces, ce qui au final en augmente la fréquence. Les plantes dotées d'un tel effet sont récoltées par les abeilles pour leur pollen ou nectar. Cité plus haut, le petit asphodèle en est l'exemple typique.

- Diminution de la fréquence d'essaimage par allongement du temps avant la sortie de l'ancienne reine. Les substances de ces plantes sont identifiées par les apiculteurs comme ayant un effet «calmant » sur les abeilles, par la diminution constatée de l'activité de la ruche voire même de sa population. D'ailleurs, certaines de ces plantes peuvent être sciemment utilisées par l'apiculteur pour dérégler le fonctionnement de la ruche. L'idée peut être par exemple défendue de renouveler la population de la ruche sans passer par l'étape d'essaimage. Une perche de laurier-rose traversant la ruche de part en part permettrait à l'apiculteur de condamner une partie du couvain alors contaminé par la toxicité de la substance issue du laurier. Celle-ci est exprimée par les apiculteurs en termes d'Wzamertume et aurait pour effet de transformer toutes les abeilles de la colonie en une population de travailleuses à qui on aurait suspendu la possibilité d'essaimer. Cette pratique est utilisée par des apiculteurs âgés qui souhaitent garder une ruche près d'eux dans la maison juste pour récolter un peu de miel sans avoir à s'occuper de l'essaimage.

- Santé et hygiène de la ruche à partir des éléments nutritifs, de l'eau et des substances végétales pour lutter contre le varroa (genre d'acarien) et autres parasites. Les besoins alimentaires de la ruche supposent un accès à des sources de pollen pour le développement du couvain et de nectar pour l'élaboration du miel, réserve énergétique de l'abeille. Les comportements de récolte observés par les apiculteurs soulignent l'importance de certaines plantes phares dans l'alimentation du couvain selon les périodes de floraison. L'eau rentre dans le même cas de figure. Élément primordial pour la régulation de plusieurs systèmes de fonctionnement de la ruche (humidification, refroidissement, hydratation), les abeilles 
vont la récolter en tout endroit accessible (d'une flaque d'eau à la rosée déposée sur le dos d'un serpent). Néanmoins, l'eau recueillie dans certaines fleurs, comme celles du grenadier, ou sur certaines feuilles, comme celles du figuier, aurait des vertus particulières. Certains latex et colles végétales sont identifiés par les apiculteurs pour être aussi directement utilisés par les abeilles sans transformation afin de lutter contre le varroa.

- Santé et hygiène de la ruche à partir de la fabrication de la propolis. Autre produit de la ruche, la propolis demande une base végétale résineuse ou tout du moins collante. Elle est utilisée de manière à recouvrir la surface intérieure de la ruche à des fins de protection anti-bactérienne et joue de ce fait un rôle essentiel dans le système sanitaire de la ruche. Dans cette catégorie se retrouvent toutes sortes de substances, du latex d'euphorbes à l'exsudat d'Ononis sans oublier le suc de séneçon, primordial pour cela. Face à l'invasion du varroa, les apiculteurs considèrent que les abeilles sont capables de réduire les effets de la maladie notamment par l'utilisation de la propolis issue de certaines plantes. D'après ces derniers, plus les latex récoltés sont « piquants » (harr), plus ils sont efficaces face au varroa. Parmi ces espèces, un certain nombre est ligneux et leur latex une réponse en cas d'agression de l'écorce, dans ce cas l'abeille blessera la surface du fruit (arganier) ou la base de la feuille (Periploca) pour le récupérer. Les espèces herbacées, quant à elles, sécrètent des exsudats accessibles directement à la surface de la plante (orge). L'apiculteur peut aussi jouer un rôle dans cette lutte. Outre le fait de déplacer les ruches et de mettre le feu au vieux matériel contaminé, il peut recourir à certaines espèces, comme le thym et l'armoise, pour enfumer les essaims et limiter ainsi l'emploi de traitements chimiques.

- Diminution de la capacité de travail des abeilles avec pour conséquence la diminution de la production de miel, ce qui n'est pas dans l'intérêt de l'apiculteur. Cependant, les abeilles semblent tout de même faire appel à ces substances qui ont un effet néfaste sur leur propre santé. Les apiculteurs identifient clairement ces espèces toxiques, fait étonnant au vu de la croyance locale selon laquelle les abeilles ponctionnent dans les plantes uniquement ce qui leur est favorable. Ils ne cachent pas que leurs connaissances n'atteignent pas l'ingéniosité de l'abeille, et répondent dans ce contexte: Allah alim, « Dieu seul sait $»$. Ainsi, pour le cas particulier du caroubier et de la sève sucrée qu'exsudent ses fruits piqués, peut-être l'abeille fait-elle la part entre ce que cette sève lui apporte de toxicité, ou de vertus et énergies.

Chacun de ces éléments va concourir à un moment de l'année au bon déroulement d'une des différentes étapes de l'évolution de la ruche. La conservation de certaines espèces lors du désherbage, par exemple, préalablement connues ainsi que leurs effets, suppose que de génération en génération, les hommes ont finalement favorisé et façonné un écosystème répondant aussi aux attentes des abeilles en termes de nutrition et de santé. Ils ont développé l'attrait apicole du territoire en valorisant ses ressources nectarifères. Les abeilles ont été progressivement invitées à adopter un régime de substances végétales réparties selon une correspondance spatiale (localisation écologique) et temporelle (floraison et développement de la ruche) du territoire. De ce point de vue, les abeilles ont été finalement totalement intégrées au territoire rural des apiculteurs. Le cycle des unes et celui des uns se calquent sur un même rythme des activités qui sont elles-mêmes tributaires du façonnement des milieux écologiques par la main des hommes avec l'aide des mandibules des abeilles.

Laménagement de la diversité végétale du territoire, clef de la performance de l'apiculture du Sud ouest marocain et de son intégration dans l'agrosystème. 
La grande hétérogénéité des unités spatiales qui forment le territoire est à la base de trois caractéristiques majeures de l'apiculture avec l'abeille saharienne dans le sud du Maroc:

- Un système sédentaire d'apiculture adapté aux besoins précis des abeilles et qui repose sur la diversité de fleurs disponibles à différentes périodes de l'année en fonction des différents espaces cultivés. Même sédentaire, l'apiculture dans le Sud marocain optimise malgré tout la diversité des matières apicoles mise à disposition pour les abeilles grâce à la diversité des unités cultivées qui fragmentent le territoire.

- Une capacité à s'adapter aux variations climatiques grâce aux potentiels déplacements des ruches d'une unité spatiale à une autre en fonction de la pluviométrie annuelle. Si pendant les saisons de sécheresse, les ruches sont déplacées dans les petites oasis, pendant les épisodes de pluies exceptionnelles, les ruches sont conservées dans les bosquets d'Opuntia à proximité du village, permettant un suivi étroit par l'apiculteur et assurant un rôle de protection contre le vol.

- Une capacité à étendre l'aire de pollinisation des arbres, et notamment des arganiers, par les abeilles en disposant les ruches à des emplacements stratégiques permettant aux abeilles de couvrir le couvert arboré des différentes unités spatiales du terroir et en premier lieu des champs.

En découpant le territoire en unités spatiales destinées à des écosystèmes différents, les Ait Ba'amran, les Haha et les Ida ou Tanan, et plus largement les paysans du Sud marocain, ont posé les conditions nécessaires au développement d'une apiculture adaptée aux aléas du climat et qui répond autant à un objectif de production de miel qu'à un objectif de tenue du territoire et de pollinisation des arbres. Plus encore, par l'emplacement dans l'espace de la ruche, l'apiculteur exerce un contrôle direct sur la reproduction de l'animal et la maîtrise des rythmes physiologiques liés à son alimentation, condition sine qua non à sa domestication. La domestication de l'abeille se réalise ainsi par l'intermédiaire du territoire, car l'apiculteur joue sur la multifonctionnalité des espaces en termes de cortège végétal pour maîtriser le cycle de vie et de production de l'abeille. Il s'appuie sur une mosaïque d'espaces de culture fournissant des matières apicoles diverses et variées ayant un impact suivi sur le comportement et le métabolisme des abeilles. La domestication de l'abeille saharienne résulte donc autant de gestes techniques appréciables sur le moment, que de longs processus d'aménagement du territoire et de sélection des espèces en tenant compte de la variable apicole. Là réside la grande différence avec l'apiculture moderne standard qui impose à l'abeille un schéma comportemental à travers l'amélioration génétique, alors qu'ici, les apiculteurs l'induisent surtout par la structure de l'habitat (la ruche et le territoire). Au lieu de sélectionner l'espèce, ils sélectionnent les milieux et agencent le territoire de manière à mieux l'intégrer. En maîtrisant et façonnant d'abord leur milieu, le paysage végétal, pour mieux agir sur leur cycle de vie et leur métabolisme, le processus de domestication des abeilles développé par l'apiculture traditionnelle au Sud du Maroc oriente sur le long terme leur comportement.

Depuis une dizaine d'années, la relation symbiotique et fortement territorialisée entre les apiculteurs marocains et l'abeille jaune saharienne est confrontée à l'intrusion des pratiques d'apiculture moderne, à partir d'un modèle standard de ruche, la ruche Langstroth, transportant avec elle des essaims d'abeilles noires venues du Nord du Maroc. Avec pour objectif unique la production de miel, ces apiculteurs méconnaissent le système de gestion des terroirs qu'ils exploitent. Aujourd'hui, les recherches manquent pour mesurer l'impact d'une telle intrusion sur le peuplement des abeilles jaunes sahariennes 
et donc sur l'agroécosystème, mais les premières observations de terrain suggèrent la présence d'hybridisme et le déplacement de l'aire de l'abeille jaune un peu plus vers le Sud. Exactement comme le propose Benjamin P. Oldroyd dans Plos Biology pour le cas américain, l'un des risques probables entraînant la disparition des populations d'abeilles réside certainement dans le changement des pratiques culturelles, et plus particulièrement dans ce cas, dans l'abandon d'une vision holiste de l'apiculture au niveau du territoire. Mais loin de disparaître, l'apiculture traditionnelle dans le Sud marocain paraît même devenir dans certains cas, le support de l'adaptation de pratiques apicoles modernes à la réalité du territoire rural.

Après avoir passé en revue ces dernières années les différentes causes possibles de la dynamique d'effondrement de certaines populations d'abeilles dans le monde, nombre de scientifiques pointent aujourd'hui l'aménagement du territoire comme principale explication et solution à ce fléau contemporain (Decourtye $\&$ al., 2006). Les jachères apicoles, dont le but est d'ensemencer d'espèces apicoles ${ }^{9}$ certaines terres agricoles laissées au repos, sont un des exemples de mise en pratique de nouvelles politiques prônant l'aménagement territorial comme clef de la lutte contre la disparition des abeilles. La culture des abeilles est redéfinie à l'image de toute production agricole, comme ayant pour objet de traiter non des êtres, mais leur milieu (Poplin, 1997, cité par Tétart, 2001 : 180). Les récentes études sur les aspects moléculaires de l'adaptation écologique des abeilles abondent dans ce sens, en soulignant la nécessité de tenir compte de l'interdépendance entre les populations animales et leur contexte agro-écologique (Parker, 2010). Ainsi, l'équilibre du rapport entre humains et abeilles résiderait moins dans des pratiques particulières, qu'il nous est de toute façon difficile à définir, que dans des agencements territoriaux. Or, l'exemple du Sud marocain nous semble apporter une démonstration infaillible sur la manière dont l'aménagement du territoire sert de matrice à l'organisation et à la pérennisation de la relation homme-abeille. Dans un contexte de gestion des aléas climatiques, puisque l'on ne peut pas s'appuyer sur une population ni sur un apport nutritif stable, on table sur la relation que l'on pérennise dans le territoire. La relation est ancrée dans la structure du territoire, dont la répartition des espaces et des espèces est pensée aussi en fonction de l'abeille. L'irrégularité climatique est ainsi compensée par la régularité de la relation inscrite dans le territoire.

Auteurs: Romain Simenel (IRD/LPED), Antonin Adam (IRD/GRED), Audrey Crousilles (Faculté de Pharmacie de Montpellier), Lahoucine Amzil (Université Mohamed V Agdal, CERGEO), Yildiz Aumeeruddy-Thomas (CNRS/ UMR CEFE 5175) 


\section{NOTES}

Photo d'ouverture: Un gardien de rucher collectif dans le Sud ouest marocain.

Sauf mention contraire toutes les photos et les schémas sont des auteurs de l'article

1. Cette proposition est applicable à toute sorte d'espèces ressources en zone aride. Il s'agit là de structurer le rapport technique à l'écosystème à partir d'une approche par la relation et non par le peuplement.

2. Lapiculture traditionnelle se définit par le recours à un corpus de savoirs et techniques transmis et complété depuis des générations et générations au sein d'un ensemble social et culturel particulier. L'apiculture traditionnelle varie ainsi d'un ensemble culturel à un autre, elle n'est pas intégrée dans un circuit commercial global mais soumise au commerce de proximité. Elle s'oppose à l'apiculture moderne qui obéit à un seul et même protocole technique standardisé aux normes imposées pour sa commercialisation sur le marché national ou international. L'exemple marocain permet toutefois de relativiser quelque peu cette dichotomie.

3. Deux stages, l'un de master 2 , ingénieur en développement rural (Antonin Adam), le second de cinquième année de Pharmacie (Audrey Crousilles), ont permis d'approfondir les données notamment sur la question de l'emplacement des ruchers et des substances récoltées par les abeilles.
4. « Les abeilles essaiment pour multiplier leur espèce. Pour cela, la vieille reine abandonne la ruche avec une grande partie des abeilles » créant ainsi un nouvel individu (Tautz et al. 2009 : 25).

5. Le déplacement des ruches dans ces lieux n'est pas considéré comme une transhumance à proprement parlé du fait qu'il soit irrégulier et partiel.

6. Les ruches modernes dites à « cadres mobiles » sont généralement constituées de « châssis en bois [carrés] dans lesquels on fixe une plaque de cire gaufrée (Tétart, 2001 : 177). Cette mobilité des cires permet de nombreuses interventions au sein de la colonie: récolte aisée, réutilisation des cires, élevage artificiel, surveillance sanitaire, etc., et peut contribuer à une amélioration des rendements.

7. Les chiffres correspondent aux polygones orange.

8. Tous les noms scientifiques et en dialecte local sont mentionnés dans le tableau 1.

9. On entend par espèces apicoles toute espèce qui va fournir des substances servant de base à des produits dont la ruche a besoin: pollen, miel, propolis,...

\section{POUR CITER CET ARTICLE}

Simenel, R., Adam, A., Crousilles A. \& al. 2015 La Domestication de l'abeille par le territoire: un exemple d'apiculture holiste dans le Sud marocain. In Dupré, L., Lasseur, J. \& R. Poccard-Chapuis Pâturages Techniques E Culture $63: 258-279$ 


\section{RÉFÉRENCES}

Bernard, J-L., Lecompte Ph., Vaissière B. 2006 Pour une gestion de l'aménagement rural alliée des abeilles. Paris: Académie d'Agriculture de France (Document abeilles et agriculture).

Decourtye A., Lecompte P., Thiebeau P. 2005 Jachères florales en zones de grandes cultures des atouts pour les pollinisateurs, Bulletin Technique Apicole 32 (1) : 29-41.

Haccour, P., 1961 Recherches sur l'abeille saharienne au Maroc, La Belgique Apicole 25 (1-2) : 13-18

Marchenay, B. 1993 Un insecte au statut incertain, Études Rurales 129-130 : 117-128.

Oldroyd Benjamin P. 2007 What's killing American honey bees? PLoS Biology, volume V, Issue 6, el68 $<$ www.plosbiology.org > Consulté en mars 2011.

Parker, R., Melathopoulos, AP., White, R., \& al. 2010 Ecological adaptation of diverse honey bee (Apis mellifera) populations, PLoS ONE 5 (6) : el1096. < doi: 10.1371/journal.pone.0011096 > Consulté en mai 2012.

Poplin, F. 1997 L'Élevage et l'animal vrai. Paris: Muséum national d'Histoire naturelle (Laboratoire d'Archéozoologie et Histoire des sociétés).

Simenel, R. 2010 L'Origine est aux frontières. Les Ait Ba'amran, un exil en terre d'arganiers (Sud-Maroc). Paris: Éditions CNRS - Maison des sciences de l'homme (Les chemins de l'ethnologie).

— 2011 Les Miels des forêts d'arganiers. Dossier thématique de l'IRD - «Suds en ligne » intitulé « Des forêts et des hommes»< http://www.mpl.ird.fr/suds-en-ligne/foret/index.html> Consulté en juin 2012.

Simenel, R., Michon, G., Auclair, \& al. 2009 L'Argan: l'huile qui cache la forêt domestique. Autrepart 50 : 51-74.

Tautz, J., Elie, Y., Heilmann, H. 2009 L'Étonnante abeille. Louvain-la-Neuve: De Boeck (Biologie animale)

Tétart, G. 2001 L'Abeille et l'apiculture. Domestication d'un animal cultivé. Techniques \& Culture 37: $173-196$.

\section{RÉSUMÉ}

La Domestication de l'abeille par le territoire. Un exemple d'apiculture holiste dans le Sud marocain. En cherchant à requalifier la domestication des abeilles à partir de pratiques plus holistes et plus ancrées dans le territoire, le propos de cet article est d'interroger la manière dont les apiculteurs du Sud Ouest marocain (D'Essaouira à Tan Tan) intègrent idéologiquement et techniquement les abeilles jaunes sahariennes dans leur monde. Les savoirfaire des apiculteurs de ces régions reposent en partie sur la manipulation de différents types d'espaces agraires, et l'apiculture est partie intégrante de l'agro-écosystème global. Bien que sédentaire, l'apiculture dans le sud du Maroc implique une grande diversité d'espaces, comprenant les falaises à chutes d'eau, les champs de céréales et d'arganiers, les zones arboricoles fermées, les zones de monoculture de figuiers de barbarie, les parcours dominés par les euphorbes et les arganiers. Chaque unité spatiale du territoire rural est le support d'un certain degré de domestication des abeilles: capture des essaims sauvages, acclimatation des ruches, production de miel, reproduction des essaims. Les savoir-faire des apiculteurs s'illustrent par la connaissance sur le comportement des abeilles (comportement alimentaire, temporalité des préférences de matières apicoles), leur métabolisme (vertus des plantes sur la vie des abeilles, leur capacité à essaimer, leur capacité à nettoyer la ruche), et la pollinisation qu'elles permettent. Les apiculteurs du Sud ouest marocain jouent ainsi sur la pluralité des espaces de cultures et des espèces qui y poussent pour intégrer l'abeille dans le territoire rural de manière holiste. Plus encore, par l'emplacement dans l'espace de la ruche, l'apiculteur exerce un contrôle direct sur la reproduction de l'animal et la maîtrise des rythmes physiologiques liés à son alimentation, condition sine qua non à sa domestication. La 
domestication se réalise ainsi par l'intermédiaire du territoire, car l'apiculteur joue sur la multifonctionnalité des espaces en termes de cortège végétal pour maîtriser le cycle de vie et de production de l'abeille. La domestication de l'abeille saharienne résulte donc autant de gestes techniques appréciables sur le moment, que de longs processus d'aménagement du territoire et de sélection des espèces en tenant compte de la variable apicole.

\section{ABSTRACT}

The Taming of the bee by the territory. An example of holistic beekeeping in the Moroccan South. By searching to redefine the domestication of bees from more holistic practices, the purpose of this article is to examine how beekeepers of South West Morocco (From Essaouira to Tan Tan) incorporate ideologically and technically yellow saharian bees in their territory. In south Morocco, beekeeping is a result of the co-evolution between the Berber groups and a unique agro-forestry system, the argan tree (Argania spinosa L.). This agro-ecosystem, shaped during innumerable generations by Berber peasants, covers some 850000 hectares in the semi-arid regions of southwest Morocco. The know-hows of beekeepers in these areas are partly based on the manipulation of various types of agrarian areas, and beekeeping is part of the global agro-ecosystem. Although sedentary, beekeeping in southern Morocco involves a wide variety of areas, including the cliffs waterfalls, agricultural fields with argan trees, arboricultural closed areas, monoculture areas of prickly pairs, pastoral areas dominated by euphorbias and argan trees. Each spatial unit of rural territory is the support of some degree of domestication of bees. Depending on the season and his needs, the beekeeper decides to place his hives in one habitat or another in order to collect new colonies, favor honey production, the health of his colonies, their rapid multiplication, or their protection in the event of extended drought. Beekeepers of South Western Morocco play on the plurality of areas of crops and species that grow there to integrate the bee in rural territory in a holistic manner. Even more, thanks to the moving location in space of the hive, the beekeeper has a direct control on the reproduction of animals and the physiological rhythms associated with its food, prerequisite to its domestication. The domestication is realized through territory because the beekeeper plays on multi functionality of spaces in terms of biodiversity to control the cycle of life and production of bees.

\section{MOTS-CLÉS}

apiculture, Maroc, abeilles, domestication, territoire, savoir-faire

\section{KEYWORDS}

beekeeping, Morocco, bees, domestication, rural territory, know-how 